\title{
The Determinants for Adolescent Glycolipid Metabolism Disorder from Prenatal Period through Adolescence: a Cohort Study
}

Xiao-Hua Liang ( $\nabla$ xiaohualiang@hospital.cqmu.edu.cn )

Clinical Epidemiology and Biostatistics Department of Children's Hospital of Chongqing Medical University

Jing-Yu Chen

Ultrasound Department of Children's Hospital of Chongqing Medical University, Chongqing

Ping Qu

Clinical Epidemiology and Biostatistics Department of Children's Hospital of Chongqing Medical University

Xian Tang

Clinical Epidemiology and Biostatistics Department of Children's Hospital of Chongqing Medical University

\section{Research Article}

Keywords: HbA1c Level, Insulin Resistance, Lipid Disorders, Metabolic Abnormalities, Quality of Life, Personality Traits

Posted Date: December 22nd, 2020

DOI: https://doi.org/10.21203/rs.3.rs-117070/v1

License: () (1) This work is licensed under a Creative Commons Attribution 4.0 International License. Read Full License 


\section{Abstract}

To explore the prevalence of and risk factors for glycolipid metabolism disorder (GLMD) from the prenatal period through childhood and adolescence. A bidirectional cohort study which was established in 2014 and followed between March 1 and July 20, 2019 were designed to evaluate the risk factors for GLMD. Two stage stratified cluster sampling was used to include participants from four communities in two counties in Chongqing. 2808 healthy children aged between 6 and 9 years in 2014 entered the cohort in 2014, and followed in 2019 with a follow-up rate of $70 \%$. 2,136 samples (aged 11.68 \pm 0.60 years) were included. The prevalence of insulin resistance (IR), prediabetes/diabetes and dyslipidemia was $21 \cdot 02 \%, 7 \cdot 19 \%$ and $21 \cdot 61 \%$, respectively. Subjects with an urban residence, no pubertal development, dyslipidemia in 2014, higher family income, higher parental education, low quality of life (QoL), and unstable neurotic and psychotic personalities had significantly elevated fasting insulin (FI) or homeostasis model assessment insulin resistance (HOMA-IR) levels; subjects with female sex, no pubertal development, dyslipidemia in 2014, obesity, gestational hypertension, maternal weight gain above Institute of Medicine guidelines, single parents, low QoL, introverted and unstable personality had increased triglyceride or triglyceride/high-density lipoprotein (HDL). Adolescent with rural residence and living convenience had higher $\mathrm{HbA} 1 \mathrm{c}$ level. We observed that low QoL status, unstable and psychotic personality traits were associated with increased GLMD risk independent of obesity, suggesting that the community intervention to improve the QoL and psychological health of children is essential.

\section{Introduction}

The increased prevalence of glycolipid metabolism disorders (GLMD) in childhood and adolescents has a well-established association with adult type 2 diabetes and cardiovascular diseases (CVDs) ${ }^{1}$. GLMD in adolescents includes insulin resistance (IR), dyslipidemia and hyperglycemia. The prevalence of IR and dyslipidemia in children and adolescents ranged from $25 \cdot 3 \%$ to $44.3 \%$ among children and adolescents according different region and different diagnosed criteria ${ }^{2,3}$. Dyslipidemia in childhood tracks into adulthood and predicts the severity of atherosclerosis in adults ${ }^{1}$. The triglyceride/high-density lipoprotein cholesterol (HDL-C) ratio was used as an IR marker for overweight and obese children ${ }^{4}$ and was also an index of GLMD. The prevalence of hyperglycemia ranged from $5 \cdot 7 \%$ to $11 \cdot 13 \%$ among children with obesity ${ }^{5}$. Despite having a lower prevalence than IR and dyslipidemia, hyperglycemia during childhood is a predictor of type 2 diabetes in adulthood ${ }^{6}$. Because childhood metabolic disorders can predict CVDs in adulthood ${ }^{4,6}$, determinants of GLMD need to be evaluated during this period. Therefore, it is important to increase our understanding of the prevalence and risk factors for GLMD during the perinatal, younger childhood and adolescence periods.

Obesity is the main cause of GLMD, and our previous study revealed that obesity is positively associated with low-density lipoprotein cholesterol (LDL-C) and TGs, but negatively correlated with HDL-C ${ }^{7}$. Moreover, previous studies have shown an increased prevalence of GLMD in individuals with a sedentary lifestyle, unhealthy dietary habits, genetic factors, exposure to higher maternal fasting blood glucose (FBG) levels in utero ${ }^{8}$ and gestational diabetes ${ }^{9}$. A study found that extraverted personality is positively correlated with triglycerides, FBG and metabolic syndrome (MS) score in adults ${ }^{10}$. However, to our knowledge, there are no studies exploring the correlation between quality of life (QoL) and GLMD or between personality traits and GLMD in children aged 10 14 years in a rural-urban cohort study. This cohort study included measures of perinatal variables, social economic status (SES), anthropometric variables and biochemical indexes in 2014 and 2019 along with QoL scores and personality characteristics in adolescents, providing an excellent opportunity to fully examine the risk factors for GLMD. Moreover, it is not clear to what extent these variables account for the variance in GLMD in the transition period from childhood to adolescence.

\section{Methods}

\section{Patient and Public Involvement}

The children and their guardians or the public were not involved in the design, or conduct, or reporting, or dissemination plans of our research.

\section{Subjects}

Two stage stratified cluster sampling was used to include participants from two counties in Chongqing that represent urban and rural areas; then, two regions per county were randomly selected, and finally, all children living in the selected region were informed and included if they satisfied the inclusion criteria. In addition, a birth-cohort in which retrospective and prospective variables were analysed to evaluate the development of glycolipid metabolism risk factors from the perinatal period through adolescence, and the perinatal variables were retrospectively collected, with physical examination conducted prospectively in 2014 and in 2019. Participants who met all the following criteria were recruited: (1) aged between six and nine years in 2014, (2) resided in the target region for more than six months, (3) did not have serious diseases (e.g., nephropathy, cardiovascular disease or cancer), and (4) obtained consent from the parents and children for participation. At baseline, all participants completed SES and family health history questionnaires and were recruited mainly from grade one and grade two from primary public-schools screening of children whose families were interested in health research. The questionnaires were administered and collected by the teachers, and the physical measures results were disseminated also by the teachers. Two thousand one hundred and thirty-six subjects (with a follow-up rate of 70\%) were ultimately included (Fig. 1) and the difference between children with follow-up and withdraw was compared in Supplementary Table S1. All work in this study was conducted in accordance with the ethical guidelines of 1964 Declaration of Helsinki and its later amendments ${ }^{11}$. The Institutional Review Board at the Children's Hospital of Chongqing Medical University gave its approval for the study (File No.: 2019-86). Informed consent was provided by all subjects and parents/guardians.

\section{Demographic variables}


Demographic information, SES (parents' occupation and education level, family income and single-parent status) were collected. Parental education level was measured on a four-point scale ( $\leq 9$ years (compulsory education in China), 9 12, 12 15 and $>15$ years), and we combined bachelor and master's degrees as there were few parents with master's degrees. Prenatal variables included maternal preconception obesity, increased body mass index of mother during pregnancy, birth with cesarean section, premature delivery (<37 weeks), birth-weight, breastfeeding, gestational hypertension (GH) and gestational diabetes. Family history of obesity and CVD was investigated. The degree of pubertal development was surveyed both by the visit of paediatrician and children or parents filling the questionnaires, which included the date of the first menstruation and first nocturnal emission, then the age was calculated.

The validity and reliability of the questionnaire were checked, and were described in detail in a previous publication ${ }^{12}$. The questionnaire was completed by the parents or guardians of the children after standard training by the research group.

\section{Physical examination}

Anthropometric measurements were conducted by well-trained pediatric nurses, and the protocol for these measurements was described in a previous publication ${ }^{12}$. Waist circumference or waist-height ratio (WHtR=waist circumference/height) was used as an alternative measure of central adiposity. Hip circumference was measured twice horizontally at the level of the pubic symphysis in the front and the gluteus maximus in the back, with the participant standing upright and with their legs together and placing their arms naturally at their sides; the mean value was used.

Blood pressure (BP) and heart rate were measured on three separate occasions with an OMRON arm-type electronic sphygmomanometer (HEM7051) using an appropriately sized BP cuff placed on the subject's right arm, with the subject in a seated position, which was described in a previous publication ${ }^{12}$.

\section{Biochemical indexes}

Venous blood (three $\mathrm{ml}$ ) was drawn from each subject in the morning after at least 12 hours of fasting and 24 hours of abstaining from high-fat and spicy foods. The biochemical markers and glycosylated hemoglobin were measured within two hours after venous blood was drawn and the protocol was introduced in detail in our previous publication ${ }^{12}$. Moreover, the ratio of TG/HDL-C was used as a parameter to assess lipid metabolism ${ }^{4}$. Siemens Centaur $\mathrm{XP}$ was used to measure fasting insulin (FI), and $\mathrm{HbA1c}$ was measured by an automatic hemoglobin analyzer (ARKRAY, Japan).

\section{Measurement of QoL and Eysenck's Personality Questionnaire (EPQ)}

The QoL questionnaire for adolescents consists of 49 items, including four factors (psychosocial function, physical and mental health, living environment and QoL satisfaction) and 13 dimensions, such as self-satisfaction, relationship of teacher and pupil, physical feeling, companionship, parenthood, physical activity ability, learning ability and attitude, self-esteem, negative emotion, attitude towards doing homework, opportunity for activity, living convenience and others (picky-eating and surroundings). The order of presentation of the 49 items was randomized. Children rated the statements on a four-point scale, and the direction of response (positive or negative) varied item by item to limit response bias. Individual item values were recoded prior to analysis so that the direction was consistent. Responses were summed and normalized according to the age-, sex-and region-specific norms of Chinese into a score ranging from 0 to 100 , normalized with a mean of 50 and SD of 10, with higher scores suggesting a better QoL ${ }^{40}$.

The Chinese version of Eysenck's personality questionnaire of China ${ }^{41}$ consisted of 88 items scored on a two-point scale (for positive items $\mathrm{NO}=0$ and YES=1), including four domains: extraversion (E) (25 items), neuroticism (N) (23 items), psychoticism (P) (18 items), and lie scales (L) (22 items). High scorers on the $\mathrm{E}$ scale indicate sociable, exciting, pleasurable, carefree, and aggressive characteristics. A higher score on the $\mathrm{N}$ scale is more likely to be a worried and moody person who tends to suffer from emotional and psychosomatic disorders. The P scale was designed to measure behaviour patterns that might be considered schizoid or psychopathic in extreme cases. The $L$ scale assesses response bias. Items in the E, N, $P$ and $L$ domains are summed and normalized to the age- and sex-specific norm of China into a score ranging from 0 to 100, normalized with a mean of 50 and SD of 10 . People are defined as middle type, tendency type and typical type if the $\mathrm{T}$ score $(\mathrm{E}$ and $\mathrm{N}$ ) ranges from $43 \cdot 3 \sim 56 \cdot 7,38 \cdot 5 \sim 43 \cdot 3$ or $56 \cdot 7 \sim 61 \cdot 5,<38 \cdot 5$ or $>61 \cdot 5$. People were considered psychotic personality if $\mathrm{T}$ score of the $\mathrm{P}$ domain $>56 \cdot 7$, and response bias was indicated when the $T$ score of the $L$ domain was $>70$.

\section{Diagnostic criteria}

Children were considered to have prediabetes/diabetes if they met at least one of the following criteria: $\mathrm{FBG} \geq 5 \cdot 6 \mathrm{mmol} / \mathrm{L}$ or $\mathrm{HbA} 1 \mathrm{c}$ level $\geq 5 \cdot 7 \%{ }^{13}$, and high lipids were defined if adolescents met one of the following criteria ${ }^{14}$ : total cholesterol (TC) $\geq 200 \mathrm{mg} / \mathrm{dL} ; \mathrm{TG} \geq 130 \mathrm{mg} / \mathrm{dL} ; \mathrm{LDL}-\mathrm{C} \geq 130 \mathrm{mg} / \mathrm{dL} ;$ or HDL-C $\leq 40$ $\mathrm{mg} / \mathrm{dL}$. Moreover, IR was indicated by HOMA-IR>3.0 based on the criteria from China ${ }^{2}$; HOMA-IR was calculated as (FI mU/L) $\times(\mathrm{FBG} \mathrm{mmol} / \mathrm{L}) / 22 \cdot 5$.

Overweight was indicated by a body mass index ${ }^{15}$ at or above the 85 th percentile and below the 95 th percentile, and obesity was defined as a BMI at or above the 95 th percentile according to the sex-specific Centers for Disease Control BMI-for-age growth charts ${ }^{15}$. The definitions of size for gestational age used the global reference for fetal-weight and birthweight percentiles ${ }^{16}$ : birth weight at or above the 90 th percentile indicated large for gestational age (LGA), and birth weight less than the 10th percentile indicated small for gestational age (SGA), using the parameters of mean birthweight at $40 \cdot 5$ weeks of $3,332 \cdot 93 \mathrm{~g}$ and a variation coefficient of $14 \cdot 36 \%$. Maternal overweight and obesity was indicated by a BMI of $24 \sim 27 \cdot 9 \mathrm{~kg} / \mathrm{m}^{2}$ and a BMI $\geq 28 \mathrm{~kg} / \mathrm{m}^{2}$, respectively; BMI $<18.5$ $\mathrm{kg} / \mathrm{m}^{2}$ was defined as a low BMI ${ }^{17}$. According to guidelines of the Institute of Medicine (IOM) for maternal pregnancy weight gain ${ }^{18}$, the recommendation is for underweight, normal weight, overweight, and obese women to gain $12.5 \sim 18.0 \mathrm{~kg}, 11.5 \sim 16.0 \mathrm{Kg}, 7 \cdot 0 \sim 11.5 \mathrm{~kg}$, and $5.0 \sim 9.0 \mathrm{~kg}$, respectively; if weight gain exceeded that range, weight gain was defined as "above IOM guidelines", and if weight gain was below that range, was defined as "below the IOM guidelines".

\section{Statistical analyses}


Differences in glycolipid metabolism indexes between two groups were assessed using Student's t-test, ANOVA was used to compare more than two groups, and post-hoc comparison was perfoemed using Student-Newman-Keuls test. Continuous variables (insulin, HOMA-IR, TG and TG/HDL) that did not satisfy a normal distribution were subjected to natural logarithmic transformation before analyses. The $\chi^{2}$ test was used to test the difference in prevalence rates of

GLMD. A generalized linear model (GLM) was used to analyze the risk factors that may impact glycolipid metabolism. To reduce the collinearity of variables, model 1 mainly included the variables measured prenatally and in 2014, and model 2 mainly included the variables measured in 2019 . Finally, model 3 included all the variables that may impact GLMD. Moreover, multivariable logistic regression was performed using diagnosed GLMD as the dependent variables with the impact factors from perinatal to adolescent, as independent variables. Adjusted $\mathrm{R}^{2}$ was calculated to locate the variance of dependent variables can be explained by independent variables. Participants with the missing responding variables were not included in the analyses. And the participants finish the follow were compared with the dropouts.

The data analysis was conducted using SAS 9.4 software (Copyright $\left(C_{2} 2020\right.$ SAS Institute Inc. Cary, NC, USA). A significant difference was defined by an a level of 0.05 .

\section{Results}

\section{General characteristics}

The general characteristics of the subjects are presented in Table 1. A total of 2,136 samples were included, with a follow up rate of $70 \cdot 0 \%$. And the difference of characteristics of childhood between participants with follow-up and withdraw were described in Supplementary Table S1. The mean age was $11 \cdot 68 \pm 0 \cdot 60$ years, and $52 \cdot 25 \%(1,116 / 2,136)$ were males. Biochemical indexes; anthropometric, perinatal, and SES variables; QoL; and personality characteristics are shown in Table 1. 
Table 1. General Characteristics of Glycolipid Metabolism Study in Adolescents.

\begin{tabular}{|c|c|}
\hline Variables & Participants included in 2019 \\
\hline Sample size & 2136 \\
\hline \multicolumn{2}{|l|}{ Region } \\
\hline Urban, No. (\%) & $1594(74 \cdot 63 \%)$ \\
\hline Rural, No. (\%) & $542(25 \cdot 37 \%)$ \\
\hline \multicolumn{2}{|l|}{ Anthropometric measures } \\
\hline Male sex, No. (\%) & $1116(52 \cdot 25 \%)$ \\
\hline Age, mean, y & $11 \cdot 68(0 \cdot 60)$ \\
\hline BMI, mean, $\mathrm{kg} / \mathrm{m}^{2}$ & $19 \cdot 10(3 \cdot 77)$ \\
\hline Height, mean, cm & $151 \cdot 78(7 \cdot 99)$ \\
\hline Weight, mean, kg & $44 \cdot 39(11 \cdot 05)$ \\
\hline Waist circumference, mean, $\mathrm{cm}$ & $66 \cdot 02(10 \cdot 14)$ \\
\hline WHtR, mean & $0.43(0.06)$ \\
\hline Hip circumference, mean, cm & $81 \cdot 80(8 \cdot 30)$ \\
\hline SBP, mean, mmHg & $105 \cdot 71(9 \cdot 56)$ \\
\hline DBP, mean, mmHg & $62 \cdot 81(6 \cdot 76)$ \\
\hline Puberty, No. (\%) & $586(31 \cdot 32 \%)$ \\
\hline \multicolumn{2}{|l|}{ Serum biochemical indexes } \\
\hline FBG, mean, mmol/L & $4 \cdot 45(0 \cdot 43)$ \\
\hline $\mathrm{TC}$, mean, mmol/L & $3 \cdot 52(0 \cdot 61)$ \\
\hline $\operatorname{tg}$, mean, mmol/L & $1.06(0.50)$ \\
\hline $\mathrm{TG}, \operatorname{mean}^{\dagger}$ & $-0 \cdot 03(0 \cdot 39)$ \\
\hline hdl-C, mean, mmol/L & $1 \cdot 44(0 \cdot 31)$ \\
\hline ldl-C, mean, mmol/L & $1 \cdot 84(0 \cdot 44)$ \\
\hline TG/ HDL-C, mean & $0 \cdot 80(0 \cdot 50)$ \\
\hline Insulin, mean, pmol/L & $83 \cdot 54(74 \cdot 85)$ \\
\hline Insulin, mean ${ }^{\dagger}$ & $4 \cdot 15(0 \cdot 73)$ \\
\hline HbA1c, mean, \% & $5 \cdot 37(0 \cdot 19)$ \\
\hline Insulin resistance index (IR), mean & $2 \cdot 40(2 \cdot 38)$ \\
\hline $\mathrm{IR}, \operatorname{mean}^{\dagger}$ & $0.57(0.74)$ \\
\hline Uric acid, mean, $\mu \mathrm{mol} / \mathrm{L}$ & $319 \cdot 64(76 \cdot 98)$ \\
\hline \multicolumn{2}{|l|}{ Perinatal measures } \\
\hline \multicolumn{2}{|l|}{ Maternal prepregnancy obesity, No. (\%) } \\
\hline Low weight & $352(21 \cdot 13 \%)$ \\
\hline Normal weight & $1158(69 \cdot 51 \%)$ \\
\hline Overweight/Obesity & $156(9 \cdot 36 \%)$ \\
\hline Increased BMI during pregnancy, mean, $\mathrm{kg} / \mathrm{m}^{2}$ & $5 \cdot 40(2 \cdot 62)$ \\
\hline \multicolumn{2}{|l|}{ Maternal weight gain, No. (\%) } \\
\hline Weight gain below IOM guidelines & $519(31 \cdot 36 \%)$ \\
\hline Within IOM guidelines & $637(38.49 \%)$ \\
\hline Weight gain above IOM guidelines & $499(30 \cdot 15 \%)$ \\
\hline Gestational age of mother, mean, $y$ & $27 \cdot 26(4 \cdot 98)$ \\
\hline Gestational age of father, mean, $y$ & $30 \cdot 23(5 \cdot 31)$ \\
\hline Gestational weeks of child, mean, weeks & $38 \cdot 86(2 \cdot 16)$ \\
\hline Birth weight, mean, g & $3271 \cdot 09(493 \cdot 62)$ \\
\hline \multicolumn{2}{|l|}{ Fatal weight of pregnancy week, No. $(\%)^{*}$} \\
\hline SGA & $133(7 \cdot 68 \%)$ \\
\hline Appropriate for gestational age & $1180(68 \cdot 13 \%)$ \\
\hline LGA & $419(24 \cdot 19)$ \\
\hline \multicolumn{2}{|l|}{ Gestational hypertension, No. (\%) ${ }^{*}$} \\
\hline No & $1967(97 \cdot 18 \%)$ \\
\hline Yes & $57(2 \cdot 82 \%)$ \\
\hline \multicolumn{2}{|l|}{ Gestational diabetes, No. (\%) ${ }^{*}$} \\
\hline No & $2001(98 \cdot 52 \%)$ \\
\hline Yes & $30(1 \cdot 48 \%)$ \\
\hline \multicolumn{2}{|l|}{ Smoke during pregnancy, No. (\%) ${ }^{*}$} \\
\hline No & $1642(87 \cdot 67 \%)$ \\
\hline Yes & $231(12 \cdot 33 \%)$ \\
\hline \multicolumn{2}{|l|}{ Birth with Caesarean operation, No. (\%) ${ }^{*}$} \\
\hline No & $700(36 \cdot 76 \%)$ \\
\hline Yes & $1204(63 \cdot 24 \%)$ \\
\hline
\end{tabular}


Income, Yuan/year, No. (\%)

$\begin{array}{ll}\sim 50,000 & 645(31 \cdot 96 \%) \\ \sim 150,000 & 853(42 \cdot 27 \%) \\ >150,000 & 520(25 \cdot 77 \%)\end{array}$

Expenditure of Food, median(IQR), Yuan/ month/person $665 \cdot 6(499 \cdot 2,998 \cdot 4)$

Marriage status, No. (\%)*

Double parents $\quad 1763(91 \cdot 82 \%)$

Single parents $\quad 157(8 \cdot 18 \%)$

Mother's education, y, No. (\%)*

$\begin{array}{ll}\sim 9 & 694(33 \cdot 27 \%) \\ \sim 12 & 726(34 \cdot 80 \%) \\ \geq 15 & 666(31 \cdot 93 \%)\end{array}$

Father's education, y, No. (\%)

$\begin{array}{ll}\sim 9 & 587(28 \cdot 15 \%) \\ \sim 12 & 750(35 \cdot 97 \%) \\ \geq 15 & 748(35 \cdot 88 \%)\end{array}$

Mother's occupation, No. (\%)*

Manager $112(5 \cdot 39 \%)$

Worker 708(34.07\%)

Technician/Researcher 65(3.13\%)

Farmer 567(27.29\%)

Other 626(30.13\%)

Father's occupation, No. (\%) ${ }^{*}$

Manager

$175(8.49 \%)$

Worker 706(34.24\%)

Technician/Researcher $\quad 177(8.58 \%)$

Farmer 573(27.79\%)

Other 431(20.90\%)

13 domains of QoL, mean

Self-satisfaction 50.05(11.34)

Relationship of teacher and pupil 53.52(10.08)

Physical feeling $\quad 49.90(10.73)$

Companionship 53.70(10.52)

Parenthood 51.35(11.34)

Physical activity ability $\quad 50 \cdot 11(10 \cdot 67)$

Learning ability and attitude $\quad 51.73(10 \cdot 20)$

Self-esteem 50.38(11.08)

Negative emotion $\quad 47 \cdot 65(11 \cdot 24)$

Attitude towards doing homework $\quad 51.41(9.07)$

Activity opportunity $\quad 54.63(9.58)$

Living convenience $54.43(7 \cdot 78)$

Other $50 \cdot 68(10 \cdot 16)$

Four factors of QoL, mean

Psychosocial factor $\quad 64 \cdot 79(10 \cdot 31)$

Physical and mental health factor 35.84(5.97)

Living environment factor 24.02(4.27)

Quality of life satisfaction factor $\quad 24 \cdot 90(4 \cdot 38)$

Total score of QoL, mean $52 \cdot 60(12 \cdot 33)$

Personality Characteristics, mean

Extraversion (E) 50.32(13.03)

Neuroticism (N) 50.37(14.67)

Psychoticism (P) 39.90(9.03)

Lie bias (L) 54.12(9.45)

BMI, body mass index; WHtR, waist-height-ratio; SBP, systemic blood pressure; DBP, diastolic blood pressure; FBG, fasting blood glucose; TC, total cholesterol; TG, triglyceride; HDL-C, high density lipoprotein cholesterol; LDL-C, low density lipoprotein cholesterol; IOM, 2009 Institute of Medicine; SGA, small for gestational age; LGA, large for gestational age; QoL, quality of life.

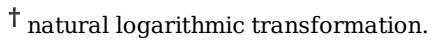

* the total sample size is unequal to 2136 in 2019 as there are missing data. 


\section{Glycolipid metabolism of children with different characteristics}

Table 2 displays the glycolipid metabolism results in adolescents. Adolescents with the characteristics of urban residence, female sex, older age, no pubertal development, dyslipidemia, obesity, low QoL score and instable personality traits had higher FI or HOMA-IR, TGs or TG/HDL than their counterparts. Whereas, $\mathrm{HbA1c}$ was higher in rural children and those with pubertal development, obesity, or maternal prepregnancy obesity than in their counterparts. In addition, TGs and/or TG/HDL were elevated in children with mother who experienced weight gain above IOM guidelines $(P<0.05)$, single-parents $(P<0 \cdot 05)$, maternal hypertension $(\mathrm{GH})$ and introversive personality compared with their counterparts $(P<0.05$ and $P=0.06)$. The levels of FI and HOMA-IR were higher in children with parents with higher education levels and family incomes than in their counterparts $(P<0 \cdot 01)$. Moreover, adolescents with psychotic personality had elevated FI and IR compared with the levels in their counterparts (all $\mathrm{P}<0 \cdot 01$ ). 
Table 2. The Glycolipid Metabolism Levels and Prevalence of Adolescent According to Perinatal and Childhood Experiences.

\begin{tabular}{|c|c|c|c|c|c|c|c|c|c|c|}
\hline \multirow[t]{3}{*}{ Variables } & \multicolumn{5}{|c|}{ Glycolipid indexes levels (median (IQR) or mean) } & \multicolumn{5}{|c|}{ Prevalence of GLMD } \\
\hline & \multirow{2}{*}{$\begin{array}{c}\text { Insulin, } \\
\text { median(IQR) }\end{array}$} & \multirow{2}{*}{$\begin{array}{c}\text { HOMA-IR, } \\
\text { median(IQR) \# }\end{array}$} & \multirow{2}{*}{$\begin{array}{c}\text { TG, } \\
\text { median(IQR) \# }\end{array}$} & \multirow{2}{*}{$\begin{array}{c}\text { TG/HDL, } \\
\text { median(IQR) }\end{array}$} & \multirow{2}{*}{$\begin{array}{l}\text { HbA1c, } \\
\text { mean\%" }\end{array}$} & HOMA-IR (>3) & \multicolumn{2}{|c|}{ Dyslipidaemia } & \multicolumn{2}{|l|}{ Prediabete } \\
\hline & & & & & & Prevalence $P$ & Prevalence & $P$ & Prevalence & \\
\hline Sample size & 2097 & 1979 & 2018 & 2018 & 932 & $416(21 \cdot 02 \%)$ & $436(21 \cdot 61 \%)$ & & $67(7 \cdot 19 \%)$ & \\
\hline \multicolumn{11}{|l|}{ Region } \\
\hline Urban & $60 \cdot 30(41 \cdot 30,99 \cdot 40)^{* *}$ & $1 \cdot 70(1 \cdot 16,2 \cdot 79)^{* *}$ & $0 \cdot 96(0 \cdot 77,1 \cdot 24)^{* *}$ & $0 \cdot 67(0 \cdot 49,0 \cdot 96)$ & $5 \cdot 34 \pm 0 \cdot 19^{* *}$ & $336(22 \cdot 86 \%)<0 \cdot 01$ & $309(20 \cdot 7 \%) 0$ & $0 \cdot 09$ & $28(6 \cdot 91 \%) 0$ & 0 \\
\hline Rural & $55 \cdot 65(35 \cdot 90,86 \cdot 50)$ & $1 \cdot 56(0 \cdot 95,2 \cdot 34)$ & $0 \cdot 90(0 \cdot 68,1 \cdot 22)$ & $0 \cdot 66(0 \cdot 46,0 \cdot 96)$ & $5 \cdot 40 \pm 0 \cdot 18$ & $80(15 \cdot 72 \%)$ & $127(24 \cdot 19 \%)$ & & $39(7 \cdot 40 \%)$ & \\
\hline \multicolumn{11}{|l|}{ Anthropometric measures } \\
\hline Sex & & & & & & & & & & \\
\hline Male & $57 \cdot 10(38 \cdot 20,93 \cdot 85)^{* *}$ & $1 \cdot 59(1 \cdot 05,2 \cdot 62)^{*}$ & $0 \cdot 91(0 \cdot 70,1 \cdot 21)^{* *}$ & $0 \cdot 65(0.46,0 \cdot 95)^{* *}$ & $5 \cdot 37 \pm 0 \cdot 19$ & $205(19 \cdot 86 \%) 0 \cdot 19$ & $223(21 \cdot 2 \%) \quad 0$ & $0 \cdot 64$ & $41(8 \cdot 17 \%)$ & 0 \\
\hline Female & $62 \cdot 00(42 \cdot 30,97 \cdot 60)$ & $1 \cdot 71(1 \cdot 16,2 \cdot 75)$ & $0 \cdot 98(0 \cdot 79,1 \cdot 26)$ & $0 \cdot 69(0.51,0.97)$ & $5 \cdot 37 \pm 0 \cdot 18$ & $211(22 \cdot 28 \%)$ & $213(22 \cdot 05 \%)$ & & $26(6 \cdot 05 \%)$ & \\
\hline Age, $y$ & & & & & & & & & & \\
\hline$\sim 10$ & $53 \cdot 50(35 \cdot 30,80 \cdot 00)^{\dagger * *}$ & $1 \cdot 53(1 \cdot 01,2 \cdot 30)^{\dagger * *}$ & $* 0 \cdot 91(0 \cdot 75,1 \cdot 17)$ & $0 \cdot 64(0 \cdot 48,0 \cdot 85)^{t^{*}}$ & $5 \cdot 39 \pm 0 \cdot 20$ & $44(15 \cdot 17 \%)<0 \cdot 01$ & $49(16 \cdot 55 \%) \quad 0$ & $0 \cdot 03$ & $7(6 \cdot 67 \%)$ & 0 \\
\hline$\sim 11$ & $57 \cdot 70(39 \cdot 90,91 \cdot 70)^{\ddagger}$ & $1 \cdot 59(1 \cdot 09,2 \cdot 59)^{\ddagger}$ & $0 \cdot 94(0 \cdot 74,1 \cdot 25)$ & $0 \cdot 67(0 \cdot 48,0 \cdot 97)^{\dagger \ddagger}$ & $5 \cdot 36 \pm 0 \cdot 19$ & $205(19 \cdot 51 \%)$ & $252(23 \cdot 53 \%)$ & & $39(7 \cdot 80 \%)$ & \\
\hline$\geq 12$ & $67 \cdot 50(43 \cdot 10,112 \cdot 40)^{\S}$ & $1 \cdot 87(1 \cdot 19,3 \cdot 13)^{\S}$ & $0 \cdot 97(0 \cdot 76,1 \cdot 26)$ & $0 \cdot 69(0.49,0 \cdot 98)^{\ddagger}$ & $5 \cdot 38 \pm 0 \cdot 18$ & $167(26 \cdot 18 \%)$ & $135(20 \cdot 74 \%)$ & & $21(6 \cdot 42 \%)$ & \\
\hline Pubertal de & & & & & & & & & & \\
\hline No & $60 \cdot 70(41 \cdot 00,99 \cdot 40)$ & $1 \cdot 71(1 \cdot 14,2 \cdot 83)^{* *}$ & $0 \cdot 96(0 \cdot 76,1 \cdot 26)^{* *}$ & $0 \cdot 68(0 \cdot 49,0 \cdot 98)^{*}$ & $5 \cdot 36 \pm 0 \cdot 20^{*}$ & $276(23 \cdot 08 \%)<0 \cdot 01$ & $262(21 \cdot 56 \%) 0$ & $0 \cdot 96$ & $34(7 \cdot 80 \%)$ & 0 \\
\hline Yes & $59 \cdot 50(39 \cdot 30,89 \cdot 40)$ & $1 \cdot 62(1 \cdot 05,2 \cdot 55)$ & $0 \cdot 89(0 \cdot 69,1 \cdot 17)$ & $0 \cdot 65(0 \cdot 45,0 \cdot 92)$ & $5 \cdot 39 \pm 0 \cdot 18$ & $96(17 \cdot 55 \%)$ & $120(21 \cdot 47 \%)$ & & $29(7 \cdot 09 \%)$ & \\
\hline Dyslipidemia, in 2014 & & & & & & & & & & \\
\hline No & $55 \cdot 4(37 \cdot 4,89 \cdot 9)^{* *}$ & $1 \cdot 59(1 \cdot 03,2 \cdot 58)^{*}$ & $0.91(0.73,1 \cdot 16)^{* *}$ & $0.62(0.46,0.82)^{* *}$ & $5 \cdot 37 \pm 0 \cdot 20$ & $138(18 \cdot 42 \%) 0 \cdot 06$ & $114(15 \cdot 64 \%)<$ & $<0 \cdot 01$ & $28(8 \cdot 75 \%) 0$ & 0 \\
\hline Yes & $59 \cdot 2(41 \cdot 5,108 \cdot 1)$ & $1 \cdot 64(1 \cdot 15,2 \cdot 99)$ & $1 \cdot 02(0 \cdot 8,1 \cdot 35)$ & $0 \cdot 78(0 \cdot 56,1 \cdot 16)$ & $5 \cdot 34 \pm 0 \cdot 21$ & $61(23 \cdot 74 \%)$ & $96(32 \cdot 65 \%)$ & & $5(4 \cdot 31 \%)$ & \\
\hline Obesity, in & & & & & & & & & & \\
\hline Normal & $54 \cdot 60(36 \cdot 90,87 \cdot 00)^{+* *}$ & $1 \cdot 52(1 \cdot 00,2 \cdot 43)^{\dagger * *}$ & $0.92(0.73,1.21)^{\dagger * *}$ & $0.64(0.46,0.90)^{\dagger * *}$ & $5 \cdot 37 \pm 0 \cdot 18$ & $200(17 \cdot 33 \%)<0 \cdot 01$ & $242(20 \cdot 46 \%)<$ & $<0 \cdot 01$ & $34(6 \cdot 19 \%) 0$ & 0 \\
\hline Overweight & $77 \cdot 30(51 \cdot 20,124 \cdot 70)^{\ddagger}$ & $2 \cdot 10(1 \cdot 42,3 \cdot 27)^{\ddagger}$ & $1 \cdot 02(0 \cdot 84,1 \cdot 26)^{\ddagger}$ & $0 \cdot 72(0 \cdot 59,1 \cdot 03)^{\ddagger}$ & $5 \cdot 37 \pm 0 \cdot 17$ & $56(29 \cdot 63 \%)$ & $44(22 \cdot 92 \%)$ & & $6(6 \cdot 19 \%)$ & \\
\hline Obesity & $89 \cdot 35(52 \cdot 80,141 \cdot 90)^{\ddagger}$ & $2 \cdot 55(1 \cdot 44,3 \cdot 91)^{\ddagger}$ & $1 \cdot 05(0 \cdot 80,1 \cdot 42)^{\ddagger}$ & $0 \cdot 79(0 \cdot 54,1 \cdot 13)^{\ddagger}$ & $5 \cdot 41 \pm 0 \cdot 20$ & $73(38 \cdot 42 \%)$ & $60(31 \cdot 41 \%)$ & & $16(11 \cdot 85 \%)$ & \\
\hline Obesity, in 2019 & & & & & & & & & & \\
\hline Normal & $53 \cdot 70(37 \cdot 20,82 \cdot 80)^{\dagger * *}$ & $1.49(1 \cdot 02,2 \cdot 33)^{\dagger * *}$ & $0.90(0.72,1.17)^{\dagger * *}$ & $0.63(0.46,0.87)^{\dagger * *}$ & $5 \cdot 36 \pm 0 \cdot 18^{+* *}$ & $245(16 \cdot 21 \%)<0 \cdot 01$ & $281(18 \cdot 33 \%)<$ & $<0 \cdot 01$ & $41(6 \cdot 35 \%)$ & 0 \\
\hline Overweight & $79 \cdot 80(54 \cdot 50,124 \cdot 65)^{\ddagger}$ & $2 \cdot 22(1 \cdot 51,3 \cdot 39)^{\ddagger}$ & $1 \cdot 10(0 \cdot 83,1 \cdot 39)^{\ddagger}$ & $0 \cdot 86(0 \cdot 61,1 \cdot 15)^{\ddagger}$ & $5 \cdot 39 \pm 0 \cdot 17^{\dagger \ddagger}$ & $89(32 \cdot 36 \%)$ & $86(30 \cdot 71 \%)$ & & $6(5 \cdot 94 \%)$ & \\
\hline Obesity & $96 \cdot 80(63 \cdot 65,150 \cdot 40)^{\S}$ & $2 \cdot 74(1 \cdot 78,4 \cdot 17)^{\S}$ & $1 \cdot 14(0 \cdot 91,1 \cdot 48)^{\ddagger}$ & $0 \cdot 90(0 \cdot 66,1 \cdot 24)^{\ddagger}$ & $5 \cdot 42 \pm 0 \cdot 20^{\ddagger}$ & $81(44 \cdot 75 \%)$ & $64(34 \cdot 78 \%)$ & & $18(10 \cdot 47 \%)$ & \\
\hline Abdominal obesity, & & & & & & & & & & \\
\hline Normal & $55 \cdot 90(37 \cdot 80,90 \cdot 20)^{* *}$ & $1.58(1.03,2 \cdot 56)^{* *}$ & $0.92(0.73,1 \cdot 20)^{* *}$ & $0.65(0.47,0.90)^{* *}$ & $5 \cdot 37 \pm 0 \cdot 18$ & $243(19 \cdot 57 \%)<0 \cdot 01$ & $265(20 \cdot 88 \%) 0$ & $0 \cdot 05$ & $39(6 \cdot 20 \%)$ & 0 \\
\hline Abdominal obesity & $87 \cdot 50(52 \cdot 40,140 \cdot 50)$ & $2 \cdot 36(1 \cdot 36,3 \cdot 85)$ & $1 \cdot 03(0 \cdot 81,1 \cdot 39)$ & $0 \cdot 79(0 \cdot 55,1 \cdot 09)$ & $5 \cdot 40 \pm 0 \cdot 19$ & $64(32 \cdot 99 \%)$ & $53(26 \cdot 9 \%)$ & & $15(11 \cdot 90 \%)$ & \\
\hline Abdominal obesity, in 201 & & & & & & & & & & \\
\hline Normal & $55 \cdot 60(38 \cdot 20,86 \cdot 60)^{* *}$ & $1 \cdot 55(1 \cdot 05,2 \cdot 47)^{* *}$ & $0.91(0.73,1 \cdot 18)^{* *}$ & $0.64(0.47,0.89)^{* *}$ & $5 \cdot 36 \pm 0 \cdot 18^{* *}$ & $287(17 \cdot 28 \%)<0 \cdot 01$ & $321(19 \cdot 04 \%)<$ & $<0 \cdot 01$ & $45(6 \cdot 27 \%)$ & 0 \\
\hline Abdominal obesity & $90 \cdot 40(58 \cdot 00,144 \cdot 70)$ & $2 \cdot 55(1 \cdot 61,4 \cdot 05)$ & $1 \cdot 17(0 \cdot 91,1 \cdot 50)$ & $0 \cdot 92(0 \cdot 66,1 \cdot 27)$ & $5 \cdot 40 \pm 0 \cdot 20$ & $127(41 \cdot 91 \%)$ & $108(35 \cdot 06 \%)$ & & $20(9 \cdot 95 \%)$ & \\
\hline Perinatal measures & & & & & & & & & & \\
\hline Maternal prepregnan & besity & & & & & & & & & \\
\hline Low weight & $62 \cdot 20(41 \cdot 70,98 \cdot 90)$ & $1 \cdot 71(1 \cdot 16,2 \cdot 78)$ & $0 \cdot 95(0 \cdot 75,1 \cdot 21)$ & $0 \cdot 66(0 \cdot 49,0 \cdot 94)$ & $5 \cdot 36 \pm 0 \cdot 20^{\dagger *}$ & $241(22 \cdot 25 \%) 0 \cdot 12$ & $221(20 \cdot 16 \%) 0$ & $0 \cdot 06$ & $36(7 \cdot 33 \%) 0$ & 0 \\
\hline Normal weight & $56 \cdot 00(39 \cdot 00,92 \cdot 50)$ & $1 \cdot 58(1 \cdot 10,2 \cdot 60)$ & $0 \cdot 96(0 \cdot 75,1 \cdot 29)$ & $0 \cdot 70(0 \cdot 48,0 \cdot 99)$ & $5 \cdot 34 \pm 0 \cdot 17^{\dagger}$ & $57(17 \cdot 87 \%)$ & $84(26 \cdot 09 \%)$ & & $3(2 \cdot 17 \%)$ & \\
\hline Overweight/Obesity & $61 \cdot 30(42 \cdot 10,109 \cdot 30)$ & $1 \cdot 77(1 \cdot 14,3 \cdot 08)$ & $1 \cdot 01(0 \cdot 75,1 \cdot 23)$ & $0 \cdot 73(0 \cdot 50,0 \cdot 96)$ & $5 \cdot 41 \pm 0 \cdot 16^{\ddagger}$ & $37(25 \cdot 52 \%)$ & $35(23 \cdot 33 \%)$ & & $9(11 \cdot 69 \%)$ & \\
\hline Maternal pregnancy weig & ht gain & & & & & & & & & \\
\hline Below IOM guidelines & $62 \cdot 40(41 \cdot 10,100 \cdot 30)$ & $1 \cdot 73(1 \cdot 15,2 \cdot 73)$ & $0.92(0 \cdot 73,1 \cdot 17)^{\dagger *}$ & $0 \cdot 65(0 \cdot 48,0 \cdot 90)$ & $5 \cdot 37 \pm 0 \cdot 18$ & $105(22 \cdot 01 \%) 0 \cdot 98$ & $96(19 \cdot 92 \%) 0$ & $0 \cdot 45$ & $16(7 \cdot 21 \%) 0$ & 0 \\
\hline Within IOM guidelines & $59 \cdot 65(39 \cdot 30,97 \cdot 80)$ & $1 \cdot 67(1 \cdot 05,2 \cdot 70)$ & $0 \cdot 97(0 \cdot 77,1 \cdot 28)^{\dagger \ddagger}$ & $0 \cdot 70(0 \cdot 49,0 \cdot 99)$ & $5 \cdot 36 \pm 0 \cdot 22$ & $129(21 \cdot 57 \%)$ & $140(23 \cdot 1 \%)$ & & $22(7 \cdot 64 \%)$ & \\
\hline Above IOM guidelines & $61 \cdot 20(43 \cdot 00,96 \cdot 80)$ & $1 \cdot 70(1 \cdot 18,2 \cdot 73)$ & $0 \cdot 99(0 \cdot 75,1 \cdot 24)^{\ddagger}$ & $0 \cdot 68(0 \cdot 51,0 \cdot 96)$ & $5 \cdot 36 \pm 0 \cdot 16$ & $100(21 \cdot 65 \%)$ & $102(21 \cdot 75 \%)$ & & $10(5 \cdot 15 \%)$ & \\
\hline Premature delivery & & & & & & & & & & \\
\hline No & $59 \cdot 90(40 \cdot 50,96 \cdot 10)$ & $1 \cdot 65(1 \cdot 13,2 \cdot 69)$ & $0 \cdot 94(0 \cdot 75,1 \cdot 22)$ & $0 \cdot 67(0 \cdot 48,0 \cdot 95)$ & $5 \cdot 36 \pm 0 \cdot 19$ & $317(21 \cdot 40 \%) 0.53$ & $316(21 \%)$ & $0 \cdot 12$ & $47(6.98 \%)$ & 0 \\
\hline Yes & $61 \cdot 50(41 \cdot 20,104 \cdot 40)$ & $1 \cdot 73(1 \cdot 12,2 \cdot 83)$ & $1 \cdot 01(0 \cdot 77,1 \cdot 26)$ & $0 \cdot 71(0.49,1 \cdot 02)$ & $5 \cdot 37 \pm 0 \cdot 18$ & $44(23 \cdot 40 \%)$ & $49(25 \cdot 93 \%)$ & & $5(6 \cdot 17 \%)$ & \\
\hline Fatal weight of pregnancy & y week & & & & & & & & & \\
\hline SGA & $60 \cdot 5(40 \cdot 4,94 \cdot 2)$ & $1 \cdot 67(1 \cdot 13,2 \cdot 61)$ & $0 \cdot 94(0 \cdot 74,1 \cdot 24)$ & $0 \cdot 68(0 \cdot 48,0 \cdot 96)$ & $5 \cdot 37 \pm 0 \cdot 19$ & $226(20 \cdot 68 \%) 0 \cdot 03$ & $236(21 \cdot 22 \%) 0$ & $0 \cdot 72$ & $35(7 \cdot 09 \%) 0$ & 0 \\
\hline Appropriate for GA & $55(39 \cdot 3,85 \cdot 1)$ & $1 \cdot 53(1 \cdot 04,2 \cdot 44)$ & $0 \cdot 89(0 \cdot 73,1 \cdot 14)$ & $0 \cdot 61(0 \cdot 44,0 \cdot 88)$ & $5 \cdot 36 \pm 0 \cdot 14$ & $20(15 \cdot 75 \%)$ & $24(18 \cdot 75 \%)$ & & $4(5 \cdot 88 \%)$ & \\
\hline LGA & $61 \cdot 3(41 \cdot 6,105 \cdot 3)$ & $1 \cdot 71(1 \cdot 15,3 \cdot 04)$ & $0 \cdot 95(0 \cdot 76,1 \cdot 21)$ & $0 \cdot 67(0.49,0 \cdot 95)$ & $5 \cdot 35 \pm 0 \cdot 20$ & $99(25 \cdot 52 \%)$ & $87(22 \cdot 14 \%)$ & & $13(7 \cdot 43 \%)$ & \\
\hline Gestational hypertension & & & & & & & & & & \\
\hline No & $59 \cdot 90(40 \cdot 40,96 \cdot 60)$ & $1 \cdot 67(1 \cdot 13,2 \cdot 70)$ & $0 \cdot 94(0 \cdot 75,1 \cdot 24)$ & $0 \cdot 67(0.48,0 \cdot 96)$ & $5 \cdot 37 \pm 0 \cdot 19$ & $384(21 \cdot 03 \%) 0 \cdot 83$ & $399(21 \cdot 45 \%) 0$ & $0 \cdot 48$ & $61(7 \cdot 30 \%) 0$ & 0 \\
\hline Yes & $59 \cdot 70(40 \cdot 50,88 \cdot 55)$ & $1 \cdot 62(1 \cdot 12,2 \cdot 61)$ & $0 \cdot 99(0 \cdot 76,1 \cdot 32)$ & $0 \cdot 70(0 \cdot 54,1 \cdot 06)$ & $5 \cdot 35 \pm 0 \cdot 20$ & $12(22 \cdot 22 \%)$ & $14(25 \cdot 45 \%)$ & & $2(8 \cdot 33 \%)$ & \\
\hline Gestati & & & & & & & & & & \\
\hline No & $59 \cdot 90(40 \cdot 20,96 \cdot 60)$ & $1 \cdot 66(1 \cdot 12,2 \cdot 70)$ & $0 \cdot 94(0 \cdot 75,1 \cdot 24)$ & $0 \cdot 67(0.48,0 \cdot 96)$ & $5 \cdot 37 \pm 0 \cdot 19$ & $393(21 \cdot 14 \%) 0 \cdot 62$ & $409(21 \cdot 59 \%) 0$ & $0 \cdot 63$ & $63(7 \cdot 35 \%) 0$ & 0 \\
\hline Yes & $73 \cdot 00(46 \cdot 60,90 \cdot 20)$ & $1 \cdot 92(1 \cdot 21,3 \cdot 00)$ & $1 \cdot 01(0 \cdot 74,1 \cdot 22)$ & $0 \cdot 70(0 \cdot 55,0 \cdot 97)$ & $5 \cdot 41 \pm 0 \cdot 17$ & $7(25 \cdot 00 \%)$ & $5(17 \cdot 86 \%)$ & & $0(0 \cdot 00 \%)$ & \\
\hline Birth with Caesarean & ration & & & & & & & & & \\
\hline No & $57 \cdot 40(38 \cdot 50,93 \cdot 90)$ & $1 \cdot 60(1 \cdot 08,2 \cdot 61)$ & $0 \cdot 93(0 \cdot 76,1 \cdot 23)$ & $0 \cdot 67(0 \cdot 49,0 \cdot 96)$ & $5 \cdot 36 \pm 0 \cdot 20$ & $132(20 \cdot 06 \%) 0 \cdot 34$ & $139(20 \cdot 81 \%) 0$ & $0 \cdot 51$ & $22(6 \cdot 92 \%) 0$ & \\
\hline Yes & $60 \cdot 60(41 \cdot 55,96 \cdot 85)$ & $1 \cdot 69(1 \cdot 15,2 \cdot 74)$ & $0 \cdot 96(0 \cdot 75,1 \cdot 25)$ & $0 \cdot 68(0.48,0 \cdot 96)$ & $5 \cdot 37 \pm 0 \cdot 18$ & $245(21 \cdot 99 \%)$ & $250(22 \cdot 12 \%)$ & & $35(7 \cdot 22 \%)$ & \\
\hline reast fe & & & & & & & & & & \\
\hline No & $65 \cdot 20(40 \cdot 50,106 \cdot 00)$ & $78(1 \cdot 13,2 \cdot 90)$ & $95(0 \cdot 8,1 \cdot 24)$ & $0.69(0.54,0.95)$ & $5 \cdot 38 \pm 0 \cdot 18$ & $43(23 \cdot 24 \%)$ & $46(24 \cdot 6 \%)$ & $0 \cdot 40$ & $5(5 \cdot 32 \%)$ & \\
\hline
\end{tabular}




\begin{tabular}{|c|c|c|c|c|c|c|c|c|}
\hline Yes & $59 \cdot 50(39 \cdot 10,96 \cdot 20)$ & $1 \cdot 62(1 \cdot 07,2 \cdot 72)$ & $0 \cdot 92(0 \cdot 72,1 \cdot 23)$ & $0.65(0.46,0.96)$ & $5 \cdot 38 \pm 0 \cdot 19$ & $207(21 \cdot 27 \%)$ & $217(21 \cdot 83 \%)$ & $42(7 \cdot 38 \%)$ \\
\hline \multicolumn{9}{|l|}{ Socioeconomic measures } \\
\hline \multicolumn{9}{|l|}{ Income, Yuan/year } \\
\hline$\sim 50,000$ & $56 \cdot 20(38 \cdot 35,90 \cdot 00)^{+* *}$ & $1.57(1.07,2 \cdot 55)^{+* *}$ & $0 \cdot 96(0 \cdot 75,1 \cdot 26)$ & $0 \cdot 68(0 \cdot 48,1 \cdot 00)$ & $5 \cdot 38 \pm 0 \cdot 20$ & $114(18 \cdot 84 \%) 0 \cdot 02$ & $146(23 \cdot 78 \%) 0 \cdot 21$ & $27(8 \cdot 39 \%) 0$ \\
\hline$\sim 150,000$ & $60 \cdot 50(40 \cdot 00,94 \cdot 80)^{\dagger}$ & $1.64(1.07,2 \cdot 64)^{\dagger}$ & $0 \cdot 93(0 \cdot 76,1 \cdot 21)$ & $0 \cdot 66(0 \cdot 48,0 \cdot 93)$ & $5 \cdot 36 \pm 0 \cdot 19$ & $161(20 \cdot 43 \%)$ & $160(19 \cdot 88 \%)$ & $24(6 \cdot 47 \%)$ \\
\hline$>150,000$ & $63 \cdot 85(42 \cdot 60,106 \cdot 45)^{\ddagger}$ & $1 \cdot 79(1 \cdot 19,3 \cdot 09)^{\ddagger}$ & $0 \cdot 94(0 \cdot 73,1 \cdot 22)$ & $0 \cdot 66(0 \cdot 48,0 \cdot 96)$ & $5 \cdot 38 \pm 0 \cdot 17$ & $122(25 \cdot 42 \%)$ & $105(21 \cdot 47 \%)$ & $12(6 \cdot 09 \%)$ \\
\hline \multicolumn{9}{|l|}{ Marriage status } \\
\hline Double parents & $59 \cdot 90(40 \cdot 40,96 \cdot 20)$ & $1 \cdot 67(1 \cdot 13,2 \cdot 70)$ & $0.94(0 \cdot 75,1 \cdot 24)^{*}$ & $0 \cdot 67(0 \cdot 48,0 \cdot 96)$ & $5 \cdot 37 \pm 0 \cdot 19$ & $349(21 \cdot 33 \%) 0 \cdot 47$ & $356(21.42 \%) 0.44$ & $56(7 \cdot 49 \%) 0$ \\
\hline Single parents & $61 \cdot 90(40 \cdot 10,91 \cdot 20)$ & $1 \cdot 67(1 \cdot 11,2 \cdot 59)$ & $1 \cdot 06(0 \cdot 80,1 \cdot 30)$ & $0 \cdot 72(0 \cdot 51,1 \cdot 05)$ & $5 \cdot 35 \pm 0 \cdot 22$ & $28(18 \cdot 79 \%)$ & $36(24 \cdot 16 \%)$ & $3(4 \cdot 17 \%)$ \\
\hline \multicolumn{9}{|l|}{ Mother's education, y } \\
\hline$\sim 9$ & $56 \cdot 10(36 \cdot 90,93 \cdot 50)^{+* *}$ & $1.59(0.97,2 \cdot 55)^{f^{* *}}$ & $0 \cdot 94(0 \cdot 73,1 \cdot 25)$ & $0 \cdot 66(0 \cdot 47,0 \cdot 97)$ & $5 \cdot 37 \pm 0 \cdot 18$ & $125(19 \cdot 20 \%) 0 \cdot 05$ & $153(22 \cdot 97 \%) 0.41$ & $24(6 \cdot 02 \%) 0$ \\
\hline$\sim 12$ & $58 \cdot 20(40 \cdot 40,92 \cdot 60)^{\dagger \ddagger}$ & $1 \cdot 62(1 \cdot 14,2 \cdot 62)^{\dagger \ddagger}$ & $0 \cdot 93(0 \cdot 76,1 \cdot 23)$ & $0 \cdot 66(0.49,0 \cdot 96)$ & $5 \cdot 38 \pm 0 \cdot 20$ & $132(19 \cdot 76 \%)$ & $145(21 \cdot 45 \%)$ & $25(8.59 \%)$ \\
\hline$\geq 15$ & $63 \cdot 85(43 \cdot 60,100 \cdot 25)^{\ddagger}$ & $1 \cdot 76(1 \cdot 22,2 \cdot 90)^{\ddagger}$ & $0.96(0 \cdot 75,1 \cdot 23)$ & $0 \cdot 68(0 \cdot 49,0 \cdot 95)$ & $5 \cdot 36 \pm 0 \cdot 20$ & $149(24 \cdot 31 \%)$ & $125(19 \cdot 94 \%)$ & $17(7 \cdot 56 \%)$ \\
\hline \multicolumn{9}{|l|}{ Father's education, y } \\
\hline$\sim 9$ & $54 \cdot 85(36 \cdot 90,90 \cdot 60)^{+* *}$ & $1.52(0.97,2 \cdot 48)^{+* *}$ & $0 \cdot 93(0 \cdot 74,1 \cdot 22)$ & $0 \cdot 65(0 \cdot 48,0 \cdot 94)$ & $5 \cdot 38 \pm 0 \cdot 19$ & $102(18.44 \%)<0.01$ & $128(22 \cdot 78 \%) 0 \cdot 44$ & $23(6 \cdot 69 \%) 0$ \\
\hline$\sim 12$ & $58 \cdot 95(40 \cdot 50,92 \cdot 00)^{\dagger}$ & $1 \cdot 62(1 \cdot 15,2 \cdot 65)^{\dagger}$ & $0 \cdot 94(0 \cdot 74,1 \cdot 22)$ & $0 \cdot 66(0 \cdot 47,0 \cdot 96)$ & $5 \cdot 38 \pm 0 \cdot 18$ & $134(19 \cdot 20 \%)$ & $157(22 \cdot 11 \%)$ & $26(8 \cdot 12 \%)$ \\
\hline$\geq 15$ & $64 \cdot 40(42 \cdot 35,105 \cdot 70)^{\ddagger}$ & $1 \cdot 80(1 \cdot 17,3 \cdot 00)^{\ddagger}$ & $0 \cdot 97(0 \cdot 76,1 \cdot 26)$ & $0 \cdot 69(0 \cdot 50,0 \cdot 97)$ & $5 \cdot 36 \pm 0 \cdot 20$ & $170(25 \cdot 00 \%)$ & $139(19.97 \%)$ & $17(6 \cdot 77 \%)$ \\
\hline \multicolumn{9}{|l|}{ Mother's occupation } \\
\hline Manager & $71 \cdot 90(43 \cdot 10,127 \cdot 80)$ & $1 \cdot 93(1 \cdot 16,3 \cdot 27)$ & $1 \cdot 00(0 \cdot 75,1 \cdot 31)$ & $0 \cdot 75(0 \cdot 48,1 \cdot 03)$ & $5 \cdot 39 \pm 0 \cdot 22$ & $29(27 \cdot 36 \%) \quad 0 \cdot 61$ & $32(29.91 \%) \quad 0.09$ & $6(11 \cdot 32 \%) \quad 0$ \\
\hline Worker & $60 \cdot 50(39 \cdot 80,92 \cdot 60)$ & $1 \cdot 67(1 \cdot 09,2 \cdot 66)$ & $0 \cdot 95(0 \cdot 74,1 \cdot 26)$ & $0 \cdot 67(0.49,0 \cdot 97)$ & $5 \cdot 38 \pm 0 \cdot 18$ & $135(20 \cdot 58 \%)$ & $133(19 \cdot 79 \%)$ & $21(6 \cdot 58 \%)$ \\
\hline Technician/Researcher & $62 \cdot 50(45 \cdot 50,92 \cdot 65)$ & $1 \cdot 73(1 \cdot 38,2 \cdot 72)$ & $1 \cdot 01(0 \cdot 7,1 \cdot 19)$ & $0 \cdot 68(0 \cdot 46,0 \cdot 84)$ & $5 \cdot 33 \pm 0 \cdot 15$ & $13(21 \cdot 31 \%)$ & $10(16 \cdot 39 \%)$ & $2(8 \cdot 00 \%)$ \\
\hline Farmer & $57 \cdot 60(39 \cdot 70,96 \cdot 70)$ & $1 \cdot 64(1 \cdot 09,2 \cdot 70)$ & $0 \cdot 93(0 \cdot 75,1 \cdot 26)$ & $0 \cdot 66(0 \cdot 48,0 \cdot 98)$ & $5 \cdot 36 \pm 0 \cdot 19$ & $110(20 \cdot 87 \%)$ & $126(23 \cdot 55 \%)$ & $20(7 \cdot 49 \%)$ \\
\hline Other & $58 \cdot 20(40 \cdot 00,96 \cdot 90)$ & $1 \cdot 61(1 \cdot 13,2 \cdot 65)$ & $0 \cdot 94(0 \cdot 76,1 \cdot 19)$ & $0 \cdot 67(0.47,0 \cdot 92)$ & $5 \cdot 37 \pm 0 \cdot 19$ & $119(20 \cdot 70 \%)$ & $121(20 \cdot 65 \%)$ & $17(6 \cdot 88 \%)$ \\
\hline \multicolumn{9}{|l|}{ Father's occupation } \\
\hline Manager & $65 \cdot 60(41 \cdot 90,96 \cdot 85)$ & $1 \cdot 81(1 \cdot 17,2 \cdot 78)$ & $0 \cdot 94(0 \cdot 77,1 \cdot 24)$ & $0 \cdot 68(0 \cdot 50,0 \cdot 91)$ & $5 \cdot 40 \pm 0 \cdot 20$ & $35(22 \cdot 15 \%) \quad 0 \cdot 09$ & $33(20.50 \%) \quad 0.55$ & $7(10 \cdot 29 \%) \quad 0$ \\
\hline Worker & $58 \cdot 65(38 \cdot 90,89 \cdot 90)$ & $1 \cdot 62(1 \cdot 05,2 \cdot 55)$ & $0 \cdot 94(0 \cdot 74,1 \cdot 23)$ & $0 \cdot 66(0 \cdot 49,0 \cdot 97)$ & $5 \cdot 37 \pm 0 \cdot 19$ & $130(19 \cdot 55 \%)$ & $136(20 \cdot 09 \%)$ & $28(8 \cdot 31 \%)$ \\
\hline Technician/Researcher & $61 \cdot 10(39 \cdot 50,116 \cdot 30)$ & $1 \cdot 69(1 \cdot 07,3 \cdot 39)$ & $0 \cdot 96(0 \cdot 73,1 \cdot 26)$ & $0 \cdot 69(0.46,0 \cdot 96)$ & $5 \cdot 34 \pm 0 \cdot 23$ & $47(29 \cdot 19 \%)$ & $32(19 \cdot 39 \%)$ & $5(8 \cdot 62 \%)$ \\
\hline Farmer & $57 \cdot 00(40 \cdot 10,94 \cdot 80)$ & $1 \cdot 62(1 \cdot 14,2 \cdot 67)$ & $0 \cdot 94(0 \cdot 75,1 \cdot 28)$ & $0 \cdot 67(0 \cdot 48,0 \cdot 98)$ & $5 \cdot 38 \pm 0 \cdot 19$ & $106(19 \cdot 78 \%)$ & $129(23 \cdot 71 \%)$ & $17(6 \cdot 46 \%)$ \\
\hline Other & $60 \cdot 05(40 \cdot 70,99 \cdot 50)$ & $1 \cdot 69(1 \cdot 16,2 \cdot 83)$ & $0 \cdot 94(0 \cdot 76,1 \cdot 17)$ & $0 \cdot 66(0 \cdot 49,0 \cdot 95)$ & $5 \cdot 37 \pm 0 \cdot 16$ & $84(21 \cdot 37 \%)$ & $89(22 \cdot 19 \%)$ & $9(5 \cdot 03 \%)$ \\
\hline \multicolumn{9}{|l|}{ Score of Quality of life } \\
\hline$\sim 40$ & $64 \cdot 70(42 \cdot 40,111 \cdot 40)^{\dagger *}$ & $1 \cdot 85(1 \cdot 15,3 \cdot 08)$ & $1 \cdot 05(0 \cdot 82,1 \cdot 34)^{\dagger * *}$ & $0.71(0.53,1.07)^{+* *}$ & $5 \cdot 37 \pm 0 \cdot 18$ & $82(25.71 \%) \quad 0.06$ & $84(26.09 \%) \quad 0.02$ & $7(4 \cdot 93 \%) \quad 0$ \\
\hline$\sim 70$ & $58 \cdot 25(40 \cdot 00,93 \cdot 65)^{\ddagger}$ & $1 \cdot 62(1 \cdot 11,2 \cdot 65)$ & $0.93(0 \cdot 74,1 \cdot 22)^{\ddagger}$ & $0.67(0.48,0.94)^{\ddagger}$ & $5 \cdot 37 \pm 0 \cdot 19$ & $293(20 \cdot 10 \%)$ & $314(21 \cdot 2 \%)$ & $52(7 \cdot 60 \%)$ \\
\hline$\geq 70$ & $55 \cdot 60(38 \cdot 20,84 \cdot 70)^{\ddagger}$ & $1 \cdot 54(1 \cdot 06,2 \cdot 43)$ & $0.85(0 \cdot 71,1 \cdot 22)^{\ddagger}$ & $0.59(0.46,0.92)^{\ddagger}$ & $5 \cdot 4 \pm 0 \cdot 18$ & $26(18 \cdot 44 \%)$ & $22(15 \cdot 07 \%)$ & $4(6 \cdot 06 \%)$ \\
\hline \multicolumn{9}{|l|}{ Extraversion (E) } \\
\hline Introversive type & $60 \cdot 10(40 \cdot 25,104 \cdot 90)$ & $1 \cdot 69(1 \cdot 12,2 \cdot 90)$ & $0 \cdot 97(0 \cdot 78,1 \cdot 27)$ & $0.71(0.51,1 \cdot 01)^{*}$ & $5 \cdot 38 \pm 0 \cdot 18$ & $111(23 \cdot 67 \%) 0 \cdot 17$ & $108(22 \cdot 69 \%) 0 \cdot 60$ & $14(5 \cdot 13 \%) 0$ \\
\hline Middle type & $60 \cdot 10(40 \cdot 40,96 \cdot 10)$ & $1 \cdot 69(1 \cdot 14,2 \cdot 66)$ & $0 \cdot 95(0 \cdot 74,1 \cdot 24)$ & $0.67(0.47,0.96)^{\dagger \neq}$ & $5 \cdot 37 \pm 0 \cdot 19$ & $166(21 \cdot 20 \%)$ & $176(22 \cdot 14 \%)$ & $33(8 \cdot 85 \%)$ \\
\hline Extrovert type & $58 \cdot 70(39 \cdot 60,90 \cdot 10)$ & $1 \cdot 59(1 \cdot 09,2 \cdot 61)$ & $0 \cdot 93(0 \cdot 74,1 \cdot 23)$ & $0.65(0.48,0.90)^{\ddagger}$ & $5 \cdot 37 \pm 0 \cdot 19$ & $121(19 \cdot 03 \%)$ & $132(20 \cdot 37 \%)$ & $17(7 \cdot 05 \%)$ \\
\hline \multicolumn{9}{|l|}{ Neuroticism (N) } \\
\hline Emotionormal & $56 \cdot 8(39 \cdot 00,89 \cdot 90)^{\dagger^{*}}$ & $1 \cdot 59(1 \cdot 11,2 \cdot 57)$ & $0.92(0 \cdot 73,1 \cdot 23)^{*}$ & $0.65(0.47,0.92)^{+*}$ & $5 \cdot 38 \pm 0 \cdot 19$ & $139(19 \cdot 39 \%) 0 \cdot 07$ & $146(20 \cdot 03 \%) 0 \cdot 29$ & $29(8 \cdot 63 \%) 0$ \\
\hline Middle type & $59 \cdot 7(39 \cdot 80,95 \cdot 00)^{\dagger \neq}$ & $1 \cdot 62(1 \cdot 10,2 \cdot 65)$ & $0 \cdot 98(0 \cdot 76,1 \cdot 25)$ & $0.70(0.50,0.97)^{\ddagger}$ & $5 \cdot 38 \pm 0 \cdot 19$ & $114(19 \cdot 93 \%)$ & $126(21 \cdot 72 \%)$ & $20(7 \cdot 63 \%)$ \\
\hline Instability & $62 \cdot 6(41 \cdot 4,108 \cdot 6)^{\ddagger}$ & $1 \cdot 78(1 \cdot 14,2 \cdot 92)$ & $0 \cdot 96(0 \cdot 77,1 \cdot 29)$ & $0 \cdot 68(0 \cdot 48,1 \cdot 01)^{\dagger \ddagger}$ & $5 \cdot 36 \pm 0 \cdot 17$ & $145(24 \cdot 21 \%)$ & $144(23 \cdot 61 \%)$ & $15(5 \cdot 19 \%)$ \\
\hline \multicolumn{9}{|l|}{ Psychoticism (P) } \\
\hline No & $59 \cdot 05(39 \cdot 8,94 \cdot 2)^{* *}$ & $1 \cdot 64(1 \cdot 10,2 \cdot 65)^{* *}$ & $0 \cdot 95(0 \cdot 75,1 \cdot 24)$ & $0 \cdot 67(0 \cdot 48,0 \cdot 96)$ & $5 \cdot 37 \pm 0 \cdot 19$ & $363(20 \cdot 47 \%) 0 \cdot 01$ & $391(21 \cdot 67 \%) 0 \cdot 99$ & $62(7 \cdot 44 \%) 0$ \\
\hline Yes & $69 \cdot 05(44 \cdot 25,137)$ & $1 \cdot 91(1 \cdot 25,3 \cdot 91)$ & $0 \cdot 96(0 \cdot 70,1 \cdot 29)$ & $0 \cdot 65(0 \cdot 47,1 \cdot 00)$ & $5 \cdot 36 \pm 0 \cdot 17$ & $35(30 \cdot 43 \%)$ & $25(21 \cdot 74 \%)$ & $2(3 \cdot 70 \%)$ \\
\hline \multicolumn{9}{|c|}{$\begin{array}{l}\text { SGA, small for gestational age; GA, gestational age; LGA, large for gestational age. } \\
\text { \# Natural logarithmic transformation were used to calculate the P value. }\end{array}$} \\
\hline \multicolumn{9}{|c|}{ I Nine hundred and thirty-two samples were included. } \\
\hline$P<0.05 ;{ }^{* *} P<0 \cdot 01$ & & & & & & & & \\
\hline
\end{tabular}

\section{Prevalence of glycolipid metabolism disorder in adolescents}

Table 2 displays the prevalence of childhood GLMD. Overall, the prevalence rates of IR, prediabetes/diabetes and dyslipidemia were $21 \cdot 02 \%, 7 \cdot 19 \%$ and $21 \cdot 61 \%$, respectively. The prevalence rates of IR and dyslipidemia were higher in children with the characteristics of older age, dyslipidemia in young childhood (6 9 years), obesity and lower QoL score than in their counterparts. Moreover, Children with urban residence, LGA status, higher family income and parental education, and unstable personality $(P=0.07)$ also had an increased prevalence of IR. The prevalence of prediabetes/diabetes was higher in children with abdominal obesity in 2014 and maternal prepregnancy obesity than in their counterparts.

Relationship of QoL and personality traits with glycolipid indexes 
The results in Table 3 showed the relationship of QoL and personality traits with glycolipid indexes after adjusting for age, sex, region and BMI. Elevated scores of self-satisfaction, relationship of teacher and pupil, physical feeling, physical activity ability, negative emotion, attitude toward doing homework, physical and mental health factor and QoL satisfaction factor were protective factors for FI, IR, TGs or TG/HDL (all P<0.05 or P<0.01), and the association of QoL satisfaction factor with TG/HDL was borderline significant $(P=0.06)$. Moreover, the score of the relationship of teacher and pupil, and psychosocial factors were protective factors for TGs and TG/HDL. However, the score of living convenience was positively correlated with HbA1c. And a higher total QoL score was negatively correlated with $\mathrm{Fl}, \mathrm{IR}, \mathrm{TGs}$ and TG/HDL (all $\mathrm{P}<0 \cdot 01$ ). High $\mathrm{P}$ and N personality scores among adolescents were positively correlated with FI and IR levels (all P<0.01). In addition, children with high E scores had decreased TG/HDL level (Table 3, all P<0.05).

Table 3. The Association of QoL and Personality Characteristics on Glycolipids Indexes Adjusting for Covariates.

\begin{tabular}{|c|c|c|c|c|c|c|c|}
\hline \multirow[t]{2}{*}{ Variables } & \multicolumn{2}{|l|}{ Insulin } & IR & TG & TG/HDL & \multicolumn{2}{|l|}{ HbA1c } \\
\hline & $\beta(95 \% \mathrm{CI})$ & $P$ & $\beta(95 \% \mathrm{CI})$ & $\beta(95 \% \mathrm{CI})$ & $\beta(95 \% \mathrm{CI})$ & $\beta(95 \% \mathrm{CI})$ & $P$ \\
\hline \multicolumn{8}{|l|}{13 domains of $Q o L$} \\
\hline Self-satisfaction & $-0 \cdot 003(-0 \cdot 006,-0 \cdot 001)$ & $0 \cdot 016$ & $-0 \cdot 003(-0 \cdot 006,-0 \cdot 001)$ & $0 \cdot 025-0 \cdot 002(-0 \cdot 004,-0 \cdot 001$ & $0 \cdot 008-0.002(-0 \cdot 004,-0 \cdot 001$ & $0 \cdot 0250 \cdot 001(-0 \cdot 001,0 \cdot 002)$ & ) $0 \cdot 370$ \\
\hline Relationship of teacher and pupil & $1-0 \cdot 003(-0 \cdot 006,0 \cdot 000)$ & 0.036 & $-0 \cdot 003(-0 \cdot 006,-0 \cdot 001)$ & $0 \cdot 049-0 \cdot 002(-0 \cdot 004,-0 \cdot 001$ & $0 \cdot 020-0.003(-0.005,-0 \cdot 001$ & $0 \cdot 018-0 \cdot 001(-0 \cdot 002,0 \cdot 001)$ & 1) $0 \cdot 390$ \\
\hline Physical feeling & $-0 \cdot 005(-0 \cdot 007,-0 \cdot 002)$ & $<0 \cdot 001$ & $-0 \cdot 004(-0 \cdot 007,-0 \cdot 002)$ & $0.002-0 \cdot 002(-0 \cdot 003,-0 \cdot 001$ & $0.025-0.003(-0.005,-0 \cdot 001$ & $0.0060 .001(0 \cdot 001,0 \cdot 002)$ & $0 \cdot 144$ \\
\hline Companionship & $-0 \cdot 001(-0 \cdot 004,0 \cdot 002)$ & 0.425 & $-0 \cdot 002(-0 \cdot 004,0 \cdot 001)$ & $0 \cdot 292-0 \cdot 001(-0 \cdot 003,0 \cdot 001)$ & $0 \cdot 124-0 \cdot 001(-0 \cdot 003,0 \cdot 001)$ & $0 \cdot 3040 \cdot 001(-0 \cdot 001,0 \cdot 001)$ & ) $0 \cdot 710$ \\
\hline Parenthood & $-0.002(-0.005,0.000)$ & $0 \cdot 071$ & $-0 \cdot 002(-0 \cdot 005,0 \cdot 001)$ & $0.126-0 \cdot 001(-0.003,0.001)$ & $0 \cdot 091-0 \cdot 002(-0 \cdot 004,0 \cdot 001)$ & $0.0820 .001(0 \cdot 000,0 \cdot 002)$ & $0 \cdot 266$ \\
\hline Physical activity ability & $-0 \cdot 003(-0.006,0 \cdot 000)$ & 0.023 & $-0 \cdot 003(-0 \cdot 006,-0 \cdot 001)$ & $0 \cdot 043-0 \cdot 003(-0 \cdot 004,-0 \cdot 001$ & $0 \cdot 002-0 \cdot 003(-0.005,-0 \cdot 001$ & $0 \cdot 0100(-0 \cdot 001,0 \cdot 001)$ & $0 \cdot 846$ \\
\hline Learning ability and attitude & $-0 \cdot 003(-0.006,0 \cdot 000)$ & $0 \cdot 044$ & $-0 \cdot 002(-0 \cdot 005,0 \cdot 001)$ & $0.102-0.001(-0 \cdot 002,0 \cdot 001)$ & $0 \cdot 368-0 \cdot 002(-0 \cdot 004,0 \cdot 000)$ & $0.0510 .001(-0.001,0 \cdot 002)$ & 0.345 \\
\hline Self-esteem & $-0 \cdot 001(-0 \cdot 003,0 \cdot 002)$ & 0.591 & $-0 \cdot 001(-0 \cdot 004,0 \cdot 002)$ & $0.588-0 \cdot 001(-0 \cdot 002,0 \cdot 001)$ & $0 \cdot 228-0 \cdot 001(-0 \cdot 003,0 \cdot 001)$ & $0.1970 .000(-0 \cdot 001,0 \cdot 001)$ & $0 \cdot 616$ \\
\hline Negative emotion & $-0 \cdot 004(-0 \cdot 006,-0 \cdot 001)$ & $0 \cdot 005$ & $-0 \cdot 003(-0 \cdot 006,-0 \cdot 001)$ & $0.032-0 \cdot 002(-0 \cdot 003,-0 \cdot 001$ & $0 \cdot 014-0.002(-0.004,-0 \cdot 001$ & $0 \cdot 0190 \cdot 001(0 \cdot 000,0 \cdot 002)$ & 0.094 \\
\hline Attitude towards doing & $-0 \cdot 006(-0 \cdot 009,-0 \cdot 003)$ & $<0 \cdot 001$ & $-0 \cdot 006(-0 \cdot 009,-0 \cdot 003)$ & $0.001-0.003(-0 \cdot 005,-0 \cdot 001$ & $0.003-0.004(-0.006,-0.002$ & $0 \cdot 001-0 \cdot 001(-0 \cdot 002,0 \cdot 001)$ & ) $0 \cdot 293$ \\
\hline \multicolumn{8}{|l|}{ homework } \\
\hline Activity opportunity & $0.001(-0.003,0 \cdot 003)$ & $0 \cdot 896$ & $0 \cdot 001(-0 \cdot 002,0 \cdot 004)$ & $0.6730 .001(-0.001,0.002)$ & $0.7240 .001(-0.002,0 \cdot 002)$ & $0.9500 .001(0.000,0 \cdot 002)$ & $0 \cdot 176$ \\
\hline Other & $-0 \cdot 003(-0 \cdot 006,-0 \cdot 001)$ & $0 \cdot 020$ & $-0 \cdot 003(-0 \cdot 006,-0 \cdot 001)$ & $0 \cdot 027-0 \cdot 001(-0 \cdot 002,0 \cdot 001)$ & $0 \cdot 805-0 \cdot 001(-0 \cdot 002,0 \cdot 002)$ & $0 \cdot 7510.001(-0 \cdot 001,0 \cdot 002)$ & 0.283 \\
\hline \multicolumn{8}{|c|}{ 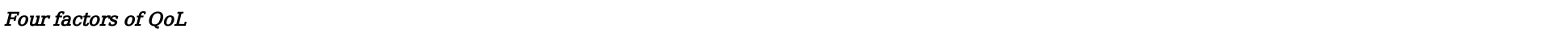 } \\
\hline Psychosocial factor & $-0 \cdot 003(-0.005,0 \cdot 000)$ & 0.063 & $-0 \cdot 002(-0 \cdot 005,0 \cdot 001)$ & $0.097-0.002(-0.003,-0.001$ & $0.041-0.002(-0.004,-0.001$ & $0.0350 .000(-0.001,0 \cdot 001)$ & 0.866 \\
\hline Physical and mental health & $-0 \cdot 010(-0 \cdot 015,-0 \cdot 005)$ & $<0 \cdot 001$ & $-0 \cdot 009(-0 \cdot 014,-0 \cdot 004)$ & $0.001-0 \cdot 004(-0 \cdot 007,-0 \cdot 002$ & $0.003-0.006(-0 \cdot 010,-0 \cdot 002$ & $0.0010 .001(-0.001,0 \cdot 003)$ & 0.236 \\
\hline \multicolumn{8}{|c|}{ ( } \\
\hline Living environment factor & $-0 \cdot 006(-0 \cdot 012,0 \cdot 001)$ & $0 \cdot 116$ & $-0 \cdot 005(-0 \cdot 013,0 \cdot 002)$ & $0.153-0.004(-0 \cdot 008,0 \cdot 001)$ & $0 \cdot 066-0 \cdot 005(-0 \cdot 010,0 \cdot 001)$ & $0 \cdot 0720.002(-0.001,0 \cdot 005)$ & 0.132 \\
\hline Quality of life satisfaction factor & $-0 \cdot 009(-0 \cdot 015,-0 \cdot 002)$ & $0 \cdot 009$ & $-0 \cdot 009(-0 \cdot 016,-0 \cdot 002)$ & $0 \cdot 014-0 \cdot 004(-0 \cdot 008,-0 \cdot 001$ & $0 \cdot 024-0 \cdot 005(-0 \cdot 01,0 \cdot 001)$ & $0.0600 .002(-0.001,0.004)$ & $0 \cdot 282$ \\
\hline Total score of $Q o L$ & $-0 \cdot 004(-0 \cdot 006,-0 \cdot 001)$ & $0 \cdot 001$ & $-0 \cdot 004(-0 \cdot 006,-0 \cdot 001)$ & $0.004-0.002(-0 \cdot 004,-0 \cdot 001$ & $0.001-0.003(-0.005,-0.001$ & $0.0010 .001(0 \cdot 000,0 \cdot 002)$ & $0 \cdot 295$ \\
\hline \multicolumn{8}{|l|}{ Personality Characteristics } \\
\hline Neuroticism (N) & $0 \cdot 003(0 \cdot 001,0 \cdot 005)$ & 0.003 & $0 \cdot 003(0 \cdot 001,0 \cdot 005)$ & $0.0140 .001(0.001,0 \cdot 002)$ & $0.0840 .001(0 \cdot 001,0 \cdot 003)$ & $0.098-0.001(-0.001,0.001)$ & $0 \cdot 223$ \\
\hline Psychoticism (P) & $0 \cdot 005(0 \cdot 001,0 \cdot 008)$ & 0.004 & $0 \cdot 005(0 \cdot 001,0 \cdot 008)$ & $0.0070 .001(-0.001,0.003)$ & $0.4700 .001(-0.002,0 \cdot 003)$ & $0 \cdot 6080.001(-0.001,0 \cdot 002)$ & $0 \cdot 781$ \\
\hline Extraversion (E) & $-0 \cdot 001(-0 \cdot 003,0 \cdot 001)$ & $0 \cdot 302$ & $-0 \cdot 002(-0 \cdot 004,0 \cdot 001)$ & $0 \cdot 211-0 \cdot 001(-0 \cdot 002,0 \cdot 001)$ & $0.105-0.002(-0.004,-0 \cdot 001$ & $0.0260 .000(-0.001,0 \cdot 001)$ & 0.773 \\
\hline
\end{tabular}
Adjusted age, sex, region and BMI in 2019.

\section{Risk factors of glycolipid metabolism indexes using a GLM}

In the GLM 1 (Table 4) (adjusted for sex, age and region), the results showed that female, living in urban areas and variables measured in 2014 (FBG, BMI, waist circumference [WC]) were risk factors for $\mathrm{FI}$ and HOMA-IR levels (all $\mathrm{P}<0.05)$, and older age was a risk factor of $\mathrm{FI}$ and IR(P<0.01); variables in 2014 (FBG, dyslipidemia, BMI) were the risk factors of TG and TG/HDL level (all P<0.01), and FBG and BMI in 2014 were risk factors for HbA1c level (Supplementary Table S2). Model 1 explained 12.43\%, 11.92\%, 6.79\%, 10.32\% and 7.06\% of the variance in FI, HOMA-IR, TGs, TG/HDL and HbA1c levels, respectively 
Table 4. The Risk Factors of Glycolipid Indexes Levels in Adolescents.

Variables Insulin, pmol/L

$\begin{array}{lll}\boldsymbol{B} & \boldsymbol{P} & \mathrm{R}^{2}\end{array}$

$\mathrm{R}^{2}$

\section{Model 1: Variables in 2014}

Sex, male vs. female

Age, y

$\begin{array}{lll}-0.136 & 0.004 \quad 12 \cdot 43 \%\end{array}$

$0.149<0.001$

$0.213 \quad 0.005$

Region, Urban vs. Rural

$-0.008 \quad 0.425$

Prepregnancy weight gain, $\mathrm{kg} / \mathrm{m}^{2}$

$\begin{array}{ll}-0.001 & 0.644\end{array}$

$0.14 \quad 0.005$

$\begin{array}{ll}0.064 & 0.238\end{array}$

$0.031 \quad 0.018$

$0.018<0.001$

HOMA-IR level

TG, mmol/L

\begin{tabular}{lll}
\hline $\boldsymbol{B}$ & $\boldsymbol{P}$ & $\mathrm{R}^{2}$ \\
\end{tabular}

FBG in 2014, mmol/L

Dyslipidemia in 2014

BMI in 2014, $\mathrm{kg} / \mathrm{m}^{2}$

Waist in 2014, cm

Gestational hypertension

Model 2: Variables in 2019

Sex, male vs. female

Age, y

Region, Urban vs. Rural

.. $. . \quad-$

$\begin{array}{ccc}-0.110 & 0.029 & 11.92 \% \\ 0.123 & 0.005 & \end{array}$

$0.123 \quad 0.005$

$0.285<0.001$

$\begin{array}{ll}-0.011 & 0 \cdot 299\end{array}$

$\begin{array}{ll}-0.002 & 0.439\end{array}$

$0.142 \quad 0.006$

$0.044 \quad 0.450$

$0.041 \quad 0.003$

$0.017 \quad 0.002$

.. ..

$\begin{array}{lll}\boldsymbol{B} & \boldsymbol{P} & \mathrm{R}^{2}\end{array}$

$\mathbf{R}^{2}$

$-0.112<0.001 \quad 6.79 \%$

$0.026 \quad 0.21$

$0.108<0.001$

..

$-0.002 \quad 0.19$

$0.080<0.001$

$0.099<0.001$

$0.019<0.001$

.. $\quad .$.

$0.096 \quad 0.20$

$-0.08<0.001 \quad 16.00 \%$

$-0.011 \quad 0.51$

$-0.04 \quad 0.15$

..

$0.001 \quad 0.89$

$0.144<0.001$

HOMA-IR level in $2019^{\dagger}$

TG/HDL in 2019

BMI in 2019, $\mathrm{kg} / \mathrm{m}^{2}$

$\begin{array}{llllll}-0.187<0.001 & 26.10 \% & -0.168 & <0.001 & 24.58 \%\end{array}$

$0.124<0.001$

$0.147 \quad 0.001$

$-0.013 \quad 0.059$

$-0.001 \quad 0.612$

$\begin{array}{ll}. . & \cdot . \\ 0.293<0.001\end{array}$

$0.033<0.001$

$0.014<0.001$

WHtR in 2019

$0.014<0.001$

Gestational hypertension

Prenatal weight gain

Below IOM guidelines

Above IOM guidelines

Puberty development

Father's Education, ref. $\leq 9 y$

$9 \sim 12$

$$
\geq 15
$$

Physical activity ability

Total score of QoL

Psychoticism personality

.

.. $\quad .$.

.. $\quad .$.

....

.. $\quad .$.

$0.085 \quad 0.040$

$0.183<0.001$

$-0.001 \quad 0.461$

$\begin{array}{ll}. . & . \cdot \\ 0.003 & 0.089\end{array}$

Model 3: Full model

Gender, male vs. female

Age, y

Region, Urban vs. Rural

$0.003 \quad 0.089$

$-0.178<0.001 \quad 28.36 \%$

$0.135<0.001$

$\begin{array}{ll}0.230 & 0.001\end{array}$

$\begin{array}{ll}-0.019 & 0.020\end{array}$

$\begin{array}{ll}-0.001 & 0.495\end{array}$

Birth weight, 50g

FBG in 2014, mmol/L

Dyslipidemia in 2014

TG/HDL in 2019

BMI in 2019, kg/m²

Waist in 2019, cm

BMI in 2014, kg/m²

HOMA-IR level in $2019^{\dagger}$

WHtR in 2019

Gestational hypertension

Prenatal weight gain

Below IOM guidelines

Above IOM guidelines

Puberty

Education, ref. $\leq 9 y$

$$
9 \sim 12
$$$$
\geq 15
$$

Physical activity ability

Psychoticism personality(P)

$0.124 \quad 0.003$

.. $\quad .$.

$0.271<0.001$

$0.045<0.001$

$0.012 \quad 0.009$

....

....

$\begin{array}{ll}. . & . . \\ . . & . .\end{array}$

..

....

.. $\quad .$.

Total score of QoL

(1)

$\begin{array}{ll}-0.001 & 0.618\end{array}$$$
0.288<0.001
$$$$
0.036<0.001
$$$$
0.014<0.001
$$$$
\text { .. } \quad . .
$$$$
\text { .. } \quad .
$$
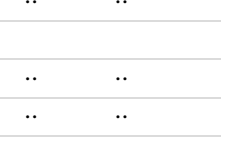

...

$0.081 \quad 0.070$

$0.177<0.001$

$-0.001 \quad 0.481$

$0.003 \quad 0.093$

$-0.159<0.001 \quad 26.33 \%$

$0.117 \quad 0.001$

$0.296<0.001$

$-0.019 \quad 0.025$

$\begin{array}{ll}-0.002 & 0.459\end{array}$

$0.125 \quad 0.005$

$\begin{array}{ll}. . & . . \\ 0.261<0.001\end{array}$

$0.049<0.001$

$0.011 \quad 0.023$

....

..$\quad$..

$. . \quad .$.

....

.. $\quad .$.

$0.08 \quad 0.145$

$0.165 \quad 0.003$

$\begin{array}{ll}-0.001 & 0.989\end{array}$

$0.006 \quad 0.012$
....

.. $\quad .$.

....

$1.150<0.001$

$0.139 \quad 0.02$

$0.074<0.001$

$0.037 \quad 0.13$

$-0.083<0.001$

.. $\quad$.

$\cdot$

$-0.002<0.001$

$\begin{array}{ll}-0.001 & 0.29\end{array}$

$-0.003<0.001$

$-0.001 \quad 0.37$

$\begin{array}{llllll}-0.107<0.001 & 17.67 \% & -0.094 & <0.001 & 19.39 \%\end{array}$

$-0.013 \quad 0.58$

$0.010 \quad 0.85$

$-0.001 \quad 0.39$

$0.070<0.001$

$0.055 \quad 0.06$

.. ..

.. $\quad$.

$\begin{array}{ll}. . & . .\end{array}$

$\begin{array}{ll}-0.008 & 0.22\end{array}$

$0.146<0.001$

$1.272<0.001$

$0.168 \quad 0.05$

$-0.009 \quad 0.76$

$\begin{array}{ll}-0.024 & 0.71\end{array}$

$-0.002 \quad 0.40$

$0.098<0.001$

$0.168<0.001$

....$$
\text { .. }
$$$$
\text { .. .. }
$$

$-0.001 \quad 0.93$

$0.17<0.001$

$1.687<0.001$

$0.174 \quad 0.13$

\section{$0.051 \quad 0.11$}

$0.059 \quad 0.08$

$0.058 \quad 0.15$

$0.078 \quad 0.07$

$-0.076 \quad 0.11$

$-0.081 \quad 0.18$

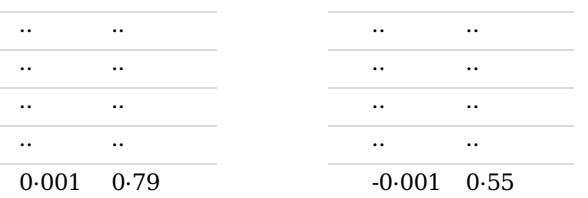

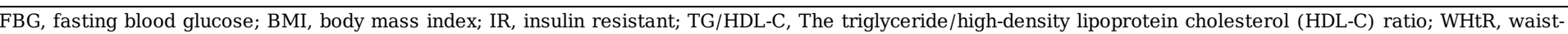
height-ratio; IOM, 2009 Institute of Medicine; QoL, quality of life.

† Natural logarithm transformation. 
The GLM (Table 4) revealed that female, older age, urban residence, and variables in 2019 (higher TG/HDL, BMI, WC, father's education $\geq 15$ years) were risk factors for $\mathrm{FI}$ and HOMA-IR level, whereas increased BMI during pregnancy was a boundary protective factor for $\mathrm{FI}$ and $\mathrm{HOMA}-\mathrm{IR}$ levels ( $\mathrm{P}=0.07$ and $\mathrm{P}=0.06$ ); HOMA-IR and WHtR in 2019, GH and maternal weight gain below IOM guidelines were risk factors for TGs and TG/HDL levels (all P<0.05), whereas puberty and total QoL score were protective factors of TGs and TG/HDL levels (all $\mathrm{P}<0.05$ or $\mathrm{P}<0.01$ ); FI and living convenience in 2019 were risk factors of HbA1c, and maternal prepregnancy obesity was a borderline risk factor of HbA1c level in model $2(P=0.07)$ (Supplementary Table S2). Model 2 explained 26.10\%, $24 \cdot 58 \%, 16 \cdot 00 \%, 17 \cdot 12 \%$ and $5 \cdot 90 \%$ of the variance in $\mathrm{FI}, \mathrm{HOMA}-\mathrm{IR}, \mathrm{TGs}, \mathrm{TG} / \mathrm{HDL}$ and $\mathrm{HbA} 1 \mathrm{c}$ levels, respectively.

Finally, the results of the full model 3 were shown in Table 4. Older age, urban area, FBG in 2014, variables in 2019 (higher TG/HDL, BMI, WC, father's education $\geq 15$ years) and high $\mathrm{P}$ scores were significantly correlated with elevated FI and IR levels (all $\mathrm{P}<0 \cdot 05$ ), while maternal prepregnancy weight gain were protective factor of FI and IR levels (all P<0.05). Variables in 2014 (FBG and dyslipidemia) and variables in 2019 (HOMA-IR and WHtR) were risk factors for TGs and TG/HDL (all P<0.05). FBG in 2014 and BMI in 2019 were risk factors for HbA1c level (Supplementary Table S2). The full model explained 28.36\%, $26 \cdot 33 \%, 17 \cdot 67 \%, 19 \cdot 39 \%$ and $12 \cdot 33 \%$ of the variance of $\mathrm{FI}, \mathrm{HOMA}-\mathrm{IR}, \mathrm{TGs}, \mathrm{TG} / \mathrm{HDL}$ and $\mathrm{HbA} 1 \mathrm{c}$ levels, respectively.

\section{Risk factors for IR, dyslipidemia and prediabetes/diabetes based on logistic regression}

The risk factors for IR, dyslipidemia and prediabetes/diabetes were analyzed by logistic regression model (Supplementary Table S3). In the IR model, older age, urban residence, FBG in 2014, BMI in 2019, father's education $\geq 15$ years and a higher $\mathrm{P}$ score had a significant impact on IR prevalence ( $P<0 \cdot 05$ ), explaining $20.09 \%$ of the variance in IR. The dyslipidemia model showed single-parents, dyslipidemia and high FBG in 2014, and BMI in 2019 were risk factors for dyslipidemia, explaining $12 \cdot 07 \%$ of the variance in dyslipidemia. The prediabetes/diabetes model revealed WHtR in 2014 was a risk factor for prediabetes/diabetes, explaining $10 \cdot 29 \%$ of the variance in prediabetes/diabetes.

\section{Discussion}

This study is the first prospective cohort study that involves QoL and personality traits, in addition to perinatal, SES and physical measurements over an average 12-year follow-up from birth to adolescence in urban-rural areas, to ascertain the prevalence of GLMD and its' potential influencing factors.

The prevalence of GLMD varies by region and age, and some variance is also attributed to different diagnostic criteria and methods. The current literature describes at least one lipid adverse level prevalence as $19 \%-25 \%$ in US children and adolescents ${ }^{8,19}$, and the prevalence of prediabetes/diabetes in other study ${ }^{5}$, was comparable with our study. An elevated prevalence of GLMD has been observed in children with obesity in cross-section study ${ }^{20}$. In this study, we found that childhood obesity is the strongest predictor of adolescent GLMD, even adjusted other risk factors. Moreover, the prevalence of HOMA-IR exceeded $44 \%$ in children who had obesity comparable with the result from Yin $\mathrm{J}$ et al. ${ }^{2}$, and the prevalence of dyslipidaemia reached $28.57 \%$ in children with abdominal obesity, suggesting that health care programmes should be conducted for children who are obesity or abdominal obesity combined with other risk factors.

In addition, a cross-sectional study revealed that elevated TG level was associated with increased HOMA-IR ${ }^{21}$, and our cohort study first found dyslipidemia and elevated fasting glucose at $6 \sim 9$ years old were independent risk factors of HOMA-IR and dyslipidemia in adolescents (10 14 years old). And adolescents with menarche or spermarche had decreased IR and lipid levels, which indicated the prepubertal stage will impact GLMD among adolescents. Whereas, the transient IR phenomenon emerging during pubertal maturation is accepted as a physiological condition ${ }^{2}$, which may be caused by an inadequate $\beta$-cell response to the decrease in insulin sensitivity ${ }^{22}$. In addition, glycolipid indexes (except HbA1c) were higher in females than in males, which coincided with the results of Interator $\mathrm{H}$. et al ${ }^{23}$, and the mechanism may be dependent on the difference in the age of prepubertal stages between males and females.

Maternal adverse perinatal experiences will impact GLMD in the offspring ${ }^{24,25}$. We found maternal prepregnancy obesity was a risk factor for irregular HbA1c level. An animal study found maternal obesity permanently alters the hypothalamic response to leptin and subsequently regulates appetite and pancreatic beta-cell physiology ${ }^{24}$, which causes maternal and off-spring changes in glycolipid levels. Moreover, our study found both maternal pregnancy weight gain above IOM guidelines and GH were risk factors for elevated off spring TGs, which coincided with the results from young adulthood ${ }^{26}$. This phenomenon can be explained by shared genes or lifestyle. However, the conclusion was controversial, as a study with a small sample size found no association between GH and lipid levels in adolescent ${ }^{27}$, this founding needs to be verified by a large cohort study. In addition, SGA and LGA correlated with elevated HOMA-IR prevalence, which coincided with other findings ${ }^{28}$. Birth-weight was correlated with nutritional status in utero, which may cause IR later in life; moreover, LGA is correlated with adolescent obesity, which is essential to IR.

SES is negatively correlated with cardiovascular disease. Our current cohort study provided further support for this concept in the adolescent population. A previous study ${ }^{29}$ revealed that the marital status of parents was the strongest socioeconomic predictor of young adult arterial stiffness, and we found the TG level was higher in single-parent adolescents. In addition, the relationship between parental education and the cardiovascular risk of adolescent is controversial, and our results showed a positive relationship between parental education or family income and FI or IR. Studies have revealed a positive correlation between parental education and childhood obesity ${ }^{30}$, and obesity was the strongest predictor of insulin sensitivity. Moreover, we observed that rural residents have lower FI, IR and TG levels, but higher HbA1c levels, which could be induced by different dietary habits, as rural children consume less fat but more carbohydrates.

Our study revealed QoL scores were mainly negatively correlated with GLMD in adolescents. Research on the relationship between QoL and GLMD is limited. According to a previous cross-sectional study that included 74 diabetic adolescents ${ }^{31}$, no significant relationship between QoL and HbA1c levels was

Page $12 / 16$ 
observed. However, a cross-sectional study found QoL scores were correlated with an increase in the components of MS, and the physical health domain of QoL had the most significant association ${ }^{32}$. In our study, we found 6 domains, four factors and the total QoL score were significantly negatively correlated with glycolipids indexes, and the effect was independent of obesity. Moreover, only the living convenience domain of QoL was positively correlated with $\mathrm{HbA1c}$, as the child has greater chance of consuming high-sugar snacks. To our knowledge, this is the first cohort study with a large sample size to explore the relationship of QoL with GLMD in adolescents.

Personality traits may be associated with GLMD, but the conclusion remains controversial, and few studies have been conducted in adult populations; no related cohort study has explored this relationship in adolescents. The results from Japanese adult ${ }^{10}$ showed the E score was positively correlated with TGs, FBG and MS components, and the P score was positively correlated with FBG. However, we found the E score in adolescents was negatively correlated with TGs and TG/HDL, suggesting the E score may be age dependent and have a different impact on lipid metabolism between adults and adolescents. And study from Evans, B. E. ${ }^{33}$ reported that extraverted adolescents have less cortisol activity, which is associated with $\mathrm{FBG}$ and $\mathrm{FI}^{34}$. Therefore, $\mathrm{E}$ personality may regulate glycolipid indexes through the hypothalamic-pituitary-adrenal axis pathway ${ }^{33}$, which may impact GLMD by regulating the reactivity of the sympathetic nervous system (SNS) ${ }^{35}$, and the SNS will have potent effects on insulin secretion and sensitivity ${ }^{36}$ and on lipid metabolism ${ }^{37}$. Higher $\mathrm{N}$ scores in adolescents would be more prone to responding more strongly to a stressor and anxiety, which could explain our results that the $\mathrm{N}$ score was positively correlated with $\mathrm{FI}$ and IR after adjusting for covariates, and $\mathrm{N}$ also correlated with lipid metabolism, which coincided with the results of study in adults ${ }^{38}$. Moreover, we found the P score was positively correlated with FI and IR, as P scores may be linked to the SNS, which may regulate insulin sensitivity ${ }^{39}$.

Our study has some limitations that should be considered when interpreting the results. First, as this was an ambispective cohort study, recall bias may exist for the prenatal variables. We checked the birth certificates to verify the birth weight, stature and gestational age. Second, data on GH and diabetes were collected using a questionnaire, and recall bias existed. However, the perinatal information both were collected both in 2014 and in 2019 independently. Finally, we were collected QoL and personality traits variables in a cross-sectional manner, which makes it difficult to draw conclusions regarding causality relationships of QoL and personality traits with GLMD.

In conclusion, the prevalence of GLMD and high glycolipid levels was elevated in adolescents with the features of obesity, maternal prepregnancy obesity, GH, SGA, LGA, single-parents status, low QoL score, and unstable or psychotic personality. SES was positively correlated with HOMA-IR. To our knowledge, this is the first study to explore the relationship of QoL and personality characteristics with glycolipid indexes in a large-sample-size cohort study of adolescents, and the correlation was significant after adjusting for covariates. Our study emphasizes the importance of reducing or controlling adiposity of pre-pregnancy mother and children, increasing QoL, developing good personality traits, emphasizing the importance of providing support for single-parent children and reducing or preventing $\mathrm{GH}$.

\section{Declarations}

\section{Data availability}

The datasets used and/or analysed during the current study are available from the corresponding author on reasonable request. Data are available from XiaoHua Liang (Clinical Epidemiology and Biostatistics Department, Children's Hospital of Chongqing Medical University, No.136 2nd Zhongshan Road, Yuzhong District, Chongqing, China, 400016; Email: xiaohualiang@hospital.cqmu.edu.cn or liangxiaohua666@sina.com).

\section{Acknowledgements}

The authors would like to acknowledge the laboratory support of the Ministry of Education Key Laboratory of Child Development and Disorders and all the staff members of the 6 elementary schools in the two regions.

\section{Author contributions}

L.X.H conceived and designed the study; C.J.Y, Q.P and T.X participated in the acquisition and management of the data; L.X.H analysed the data; L.X.H wrote the paper and all authors revised the manuscript; and all authors critically reviewed and approved the final paper.

\section{Funding}

This work was supported by National Key Research and Development Project of the Ministry of Science and Technology of the People's Republic of China (No.2017YFC0211705), Young Scientists Fund Program of the National Natural Science Foundation of China (No.81502826), School Funded Project of Chongqing Medical University (No.CQMUNCP0204), Young Scientists Fund Program of the Education Commission of Chongqing (No.KJQN201900443), General Program of the China Postdoctoral Science Foundation (No.2014M562289), and Chongqing Postdoctoral Research Funded Project (No.Xm2014129). The funders had no role in the study design, the data collection and analysis, the decision to publish, or the preparation of the manuscript.

\section{Competing interest}

The authors declare no competing interests.

\section{Additional information}

Correspondence and requests for materials should be addressed to L.X.H 


\section{References}

1 Koskinen, J. S. et al. Childhood risk factors and carotid atherosclerotic plaque in adulthood: The Cardiovascular Risk in Young Finns Study. Atherosclerosis 293, 18-25, doi:10.1016/j.atherosclerosis.2019.11.029 (2019).

2 Yin, J. et al. Insulin resistance determined by Homeostasis Model Assessment (HOMA) and associations with metabolic syndrome among Chinese children and teenagers. Diabetology \& metabolic syndrome 5, 71, doi:10.1186/1758-5996-5-71 (2013).

3 Perak, A. M. et al. Trends in Levels of Lipids and Apolipoprotein B in US Youths Aged 6 to 19 Years, 1999-2016. Jama 321, 1895-1905, doi:10.1001/jama.2019.4984 (2019).

4 Iwani, N. A. et al. Triglyceride to HDL-C Ratio is Associated with Insulin Resistance in Overweight and Obese Children. Scientific reports 7, 40055, doi:10.1038/srep40055 (2017).

5 Hagman, E. et al. Impaired fasting glucose prevalence in two nationwide cohorts of obese children and adolescents. International journal of obesity (2005) 38, 40-45, doi:10.1038/ijo.2013.124 (2014).

6 Hagman, E., Danielsson, P., Brandt, L., Ekbom, A. \& Marcus, C. Association between impaired fasting glycaemia in pediatric obesity and type 2 diabetes in young adulthood. Nutrition \& diabetes 6, e227, doi:10.1038/nutd.2016.34 (2016).

7 Liang, X., He, Y., Chen, M., Ping, Q. \& Chen, J. The association of lecithin retinol acyltransferase and the 25(OH)D receptor with pediatric overweight and obesity. European journal of clinical nutrition, doi:10.1038/s41430-019-0422-0 (2019).

8 Scholtens, D. M. et al. Hyperglycemia and Adverse Pregnancy Outcome Follow-up Study (HAPO FUS): Maternal Glycemia and Childhood Glucose Metabolism. Diabetes care 42, 381-392, doi:10.2337/dc18-2021 (2019).

9 Lowe, W. L., Jr. et al. Hyperglycemia and Adverse Pregnancy Outcome Follow-up Study (HAPO FUS): Maternal Gestational Diabetes Mellitus and Childhood Glucose Metabolism. Diabetes care 42, 372-380, doi:10.2337/dc18-1646 (2019).

10 Ohseto, H. et al. Relationships among personality traits, metabolic syndrome, and metabolic syndrome scores: The Kakegawa cohort study. Journal of psychosomatic research 107, 20-25, doi:10.1016/j.jpsychores.2018.01.013 (2018).

11 World Medical Association declaration of Helsinki. Recommendations guiding physicians in biomedical research involving human subjects. Jama 277, 925-926 (1997).

12 Liang, X., Xiao, L., Luo, Y. \& Xu, J. Prevalence and risk factors of childhood hypertension from birth through childhood: a retrospective cohort study. Journal of human hypertension, doi:10.1038/s41371-019-0282-z (2019).

13 American Diabetes, A. Diagnosis and classification of diabetes mellitus. Diabetes care 35 Suppl 1, S64-71, doi:10.2337/dc12-s064 (2012).

14 Expert Panel on Integrated Guidelines for Cardiovascular, H., Risk Reduction in, C., Adolescents, National Heart, L. \& Blood, I. Expert panel on integrated guidelines for cardiovascular health and risk reduction in children and adolescents: summary report. Pediatrics 128 Suppl 5, S213-256, doi:10.1542/peds.2009-2107C (2011).

15 Division of Nutrition, P. A., and Obesity, National Center for Chronic Disease Prevention and Health Promotion. Using the CDC BMI-for-age Growth Charts to Assess Growth in the United States Among Children and Teens Aged 2 Years to 20 Years. Available from:

http://www.cdc.gov/growthcharts/clinical_charts.htm.

16 Mikolajczyk, R. T. et al. A global reference for fetal-weight and birthweight percentiles. Lancet (London, England) 377, 1855-1861, doi:10.1016/S01406736(11)60364-4 (2011).

$17 \mathrm{Hu}, \mathrm{L}$. et al. Prevalence of overweight, obesity, abdominal obesity and obesity-related risk factors in southern China. PloS one 12, e0183934, doi:10.1371/journal.pone.0183934 (2017).

18 Johnson, J. et al. Pregnancy outcomes with weight gain above or below the 2009 Institute of Medicine guidelines. Obstetrics and gynecology 121, 969-975, doi:10.1097/AOG.0b013e31828aea03 (2013).

19 Kit, B. K. et al. Prevalence of and trends in dyslipidemia and blood pressure among US children and adolescents, 1999-2012. JAMA pediatrics 169, 272-279, doi:10.1001/jamapediatrics.2014.3216 (2015).

$20 \mathrm{Yi}, \mathrm{K}$. H. et al. Prevalence of insulin resistance and cardiometabolic risk in Korean children and adolescents: a population-based study. Diabetes research and clinical practice 103, 106-113, doi:10.1016/j.diabres.2013.10.021 (2014).

21 Simental-Mendia, L. E., Castaneda-Chacon, A., Rodriguez-Moran, M., Aradillas-Garcia, C. \& Guerrero-Romero, F. Relationship between elevated triglyceride levels with the increase of HOMA-IR and HOMA-beta in healthy children and adolescents with normal weight. European journal of pediatrics 174 597-605, doi:10.1007/s00431-014-2426-z (2015). 

clinical endocrinology and metabolism 88, 1417-1427, doi:10.1210/jc.2002-021442 (2003).

23 Interator, H. et al. Sex, Ethnicity, and Socioeconomic Status Affect on Israeli Pediatric Lipid Testing Despite Equality in National Healthcare Services. The Israel Medical Association journal : IMAJ 21, 369-375 (2019).

24 Patel, N., Pasupathy, D. \& Poston, L. Determining the consequences of maternal obesity for offspring health. Experimental physiology 100, 1421-1428, doi:10.1113/EP085132 (2015).

25 Gomes, D. et al. Late-pregnancy dysglycemia in obese pregnancies after negative testing for gestational diabetes and risk of future childhood overweight: An interim analysis from a longitudinal mother-child cohort study. PLoS medicine 15, e1002681, doi:10.1371/journal.pmed.1002681 (2018).

26 Alsnes, I. V. et al. Hypertension in Pregnancy and Offspring Cardiovascular Risk in Young Adulthood: Prospective and Sibling Studies in the HUNT Study (Nord-Trondelag Health Study) in Norway. Hypertension (Dallas, Tex. : 1979) 69, 591-598, doi:10.1161/HYPERTENSIONAHA.116.08414 (2017).

27 Fraser, A., Nelson, S. M., Macdonald-Wallis, C., Sattar, N. \& Lawlor, D. A. Hypertensive disorders of pregnancy and cardiometabolic health in adolescent offspring. Hypertension (Dallas, Tex. : 1979) 62, 614-620, doi:10.1161/HYPERTENSIONAHA.113.01513 (2013).

28 Dabelea, D. et al. Birth weight, type 2 diabetes, and insulin resistance in Pima Indian children and young adults. Diabetes care 22, 944-950, doi:10.2337/diacare.22.6.944 (1999).

29 Liang, X. et al. Determinants of pulse wave velocity trajectories from youth to young adulthood: the Georgia Stress and Heart Study. Journal of hypertension, doi:10.1097/HJH.0000000000001933 (2018).

30 Muthuri, S. K. et al. Relationships between Parental Education and Overweight with Childhood Overweight and Physical Activity in 9-11 Year Old Children: Results from a 12-Country Study. PloS one 11, e0147746, doi:10.1371/journal.pone.0147746 (2016).

31 Afshar M, M. R., Mohammadi. Investigating the relationship between quality of life, self-care capability and $\mathrm{HbA} 1 \mathrm{c}$ level in diabetic adolescents. Journal of Kashan University of Medical Sciences 18, 8 (2014).

32 Amiri, P. et al. Factors Affecting Gender Differences in the Association between Health-Related Quality of Life and Metabolic Syndrome Components: Tehran Lipid and Glucose Study. PloS one 10, e0143167, doi:10.1371/journal.pone.0143167 (2015).

33 Evans, B. E. et al. Neuroticism and extraversion in relation to physiological stress reactivity during adolescence. Biological psychology 117 , $67-79$, doi:10.1016/j.biopsycho.2016.03.002 (2016).

34 Park, S. B., Blumenthal, J. A., Lee, S. Y. \& Georgiades, A. Association of cortisol and the metabolic syndrome in Korean men and women. Journal of Korean medical science 26, 914-918, doi:10.3346/jkms.2011.26.7.914 (2011).

35 Koolhaas, J. M., de Boer, S. F., Buwalda, B. \& van Reenen, K. Individual variation in coping with stress: a multidimensional approach of ultimate and proximate mechanisms. Brain, behavior and evolution 70, 218-226, doi:10.1159/000105485 (2007).

36 Morton, G. J. et al. Evidence That the Sympathetic Nervous System Elicits Rapid, Coordinated, and Reciprocal Adjustments of Insulin Secretion and Insulin Sensitivity During Cold Exposure. Diabetes 66, 823-834, doi:10.2337/db16-1351 (2017).

37 Geerling, J. J. et al. Sympathetic nervous system control of triglyceride metabolism: novel concepts derived from recent studies. Journal of lipid research 55, 180-189, doi:10.1194/jlr.R045013 (2014).

38 van Doornen, L. J. \& Orlebeke, K. F. Stress, personality and serum-cholesterol level. Journal of human stress 8, 24-29, doi:10.1080/0097840X.1982.9936116 (1982).

39 Laederach-Hofmann, K., Kupferschmid, S. \& Mussgay, L. Links between body mass index, total body fat, cholesterol, high-density lipoprotein, and insulin sensitivity in patients with obesity related to depression, anger, and anxiety. The International journal of eating disorders 32 , 58-71, doi:10.1002/eat.10063 (2002).

40 WU Han-rong, L. P.-I., MENG Heng. Norn, Reliability and Validity of Children and Adolescents' QOL scale. Chinese Journal of School Health 27, 4 (2006).

41 Yufeng Yang. Rating Scales For Children's Developmental Behavior and Mental Health. People's Medical Publishing House co., I. Rating Scales For Children's Developmental Behavior and Mental Health. . People's Medical Publishing House co., Itd. (2016).

\section{Figures}




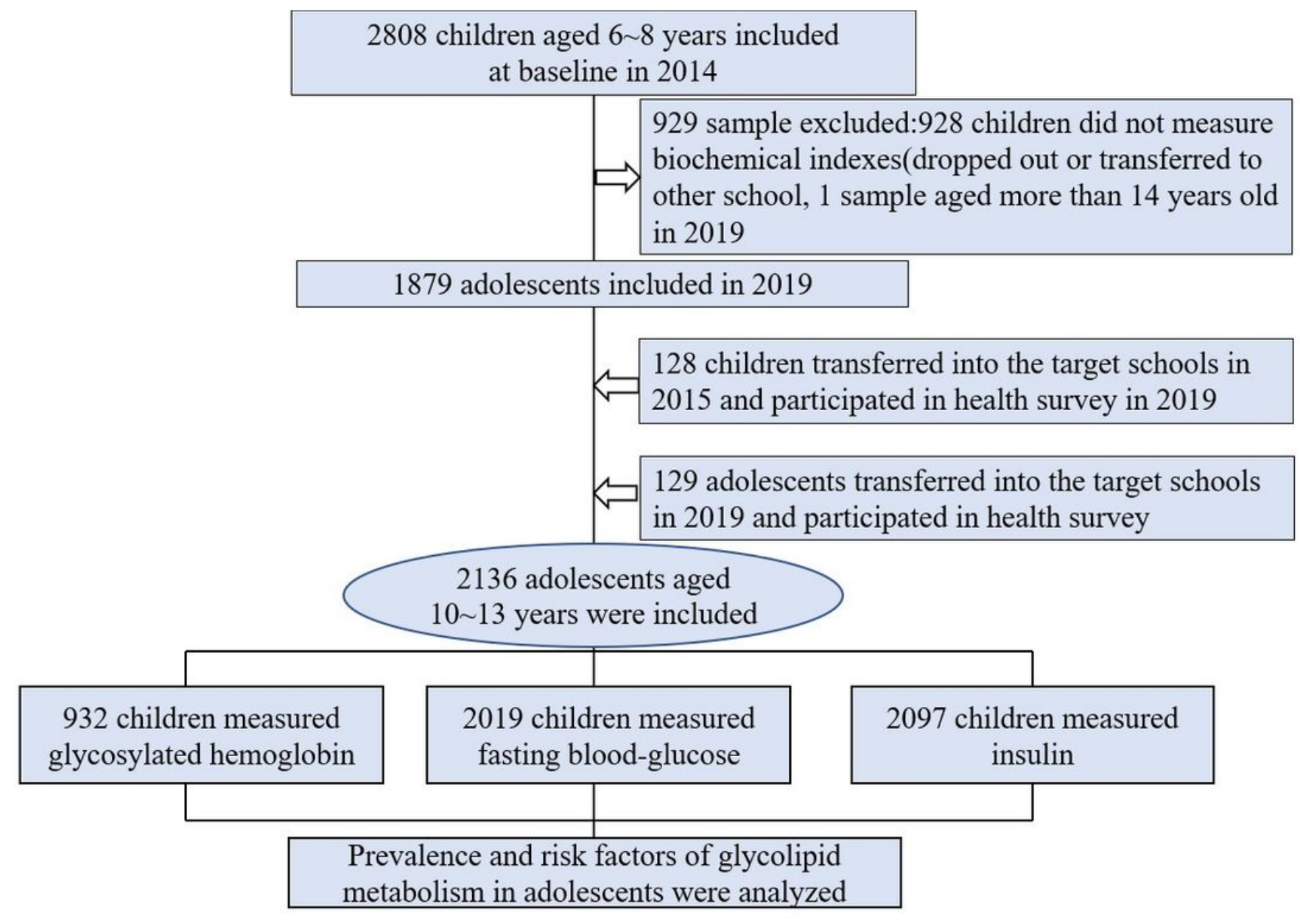

Figure 1

Subjects inclusion process

\section{Supplementary Files}

This is a list of supplementary files associated with this preprint. Click to download.

- SupplementaryTableS1S3.pdf 